\title{
ANALYTIC SHEAVES OF LOCAL COHOMOLOGY
}

\author{
BY \\ YUM-TONG SIU
}

In this paper we are interested in the following problem: Suppose $V$ is an analytic subvariety of a (not necessarily reduced) complex analytic space $X, \mathscr{F}$ is a coherent analytic sheaf on $X-V$, and $\theta: X-V \rightarrow X$ is the inclusion map. When is $\theta_{q}(\mathscr{F})$ coherent (where $\theta_{q}(\mathscr{F})$ is the $q$ th direct image of $\mathscr{F}$ under $\theta$ )?

The case $q=0$ is very closely related to the problem of extending $\mathscr{F}$ to a coherent analytic sheaf on $X$. This problem of extension has already been dealt with in Frisch-Guenot [1], Serre [9], Siu [11]-[14], Thimm [17], and Trautmann [18]-[20]. So, in our investigation we assume that $\mathscr{F}$ admits a coherent analytic extension on all of $X$.

In reponse to a question of Serre $[9$, p. 366], Tratumann has obtained the following in [21]:

THEOREM T. Suppose $V$ is an analytic subvariety of a complex analytic space $X$, $q$ is a nonnegative integer, and $\mathscr{F}$ is a coherent analytic sheaf on $X$. Let $\theta: X-V \rightarrow X$ be the inclusion map. Then a sufficient condition for $\theta_{0}(\mathscr{F} \mid X-V), \ldots, \theta_{q}(\mathscr{F} \mid X-V)$ to be coherent is: for $x \in V$ there exists an open neighborhood $U$ of $x$ in $X$ such that $\operatorname{codh}(\mathscr{F} \mid U-V) \geqq \operatorname{dim}_{x} V+q+2$.

Trautmann's sufficiency condition is in general not necessary, as is shown by the following example. Let $X=C^{3}, V=\left\{z_{1}=z_{2}=0\right\}, q=0$, and $\mathscr{F}=$ the analytic idealsheaf of $\left\{z_{2}=z_{3}=0\right\}$. $\theta_{0}(\mathscr{F} \mid X-V)=\mathscr{F}$ is coherent on $X$. However, codh $\mathscr{F}=2$ at $(a, 0,0)$ for all $a$. Hence there exists no neighborhood $U$ of $(0,0,0)$ such that $\operatorname{codh}(\mathscr{F} \mid U-V) \geqq 3$.

In this paper we derive a necessary and sufficient condition for the coherence of $\theta_{0}(\mathscr{F} \mid X-V), \ldots, \theta_{q}(\mathscr{F} \mid X-V)$. This necessary and sufficient condition is then applied to give an affirmative answer to a question raised by Serre [9, pp. 373-374].

To state the main results, we introduce the following notations: If $\mathscr{F}$ is a coherent analytic sheaf on a complex analytic space $X$, then $S_{k}(\mathscr{F})$ denotes the analytic subvariety $\left\{x \in X \mid \operatorname{codh} \mathscr{F}_{x} \leqq k\right\}$, [6, Satz 5, p. 81]. If $D$ is an open subset of $X$, then $\bar{S}_{k}(\mathscr{F} \mid D)$ denotes the topological closure of $S_{k}(\mathscr{F} \mid D)$ in $X$. If $V$ is an analytic subvariety of $X$, then $\mathscr{H}_{V}^{k}(\mathscr{F})$ denotes the sheaf defined by the presheaf $U \mapsto H_{V}^{k}(U, \mathscr{F})$, where, $H_{V}^{k}(U, \mathscr{F})$ is the $k$-dimensional cohomology group of $U$ with coefficients in $\mathscr{F}$ and supports in $V$. If $X, \mathscr{F}$, and $V$ are complex algebraic instead of analytic, we use the same notations and $\mathscr{F}^{h}$ denotes the coherent analytic sheaf canonically associated with $\mathscr{F}$.

Received by the editors April 22, 1969. 
The main results are the following two theorems:

THEOREM A. Suppose $V$ is an analytic subvariety of a complex analytic space $(X, \mathscr{H}), q$ is a nonnegative integer, and $\mathscr{F}$ is a coherent analytic sheaf on $X$. Let $\theta: X-V \rightarrow X$ be the inclusion map. Then the following three statements are equivalent:

(i) $\theta_{0}(\mathscr{F} \mid X-V), \ldots, \theta_{q}(\mathscr{F} \mid X-V)$ (or equivalently $\left.\mathscr{H}_{V}^{0}(\mathscr{F}), \ldots, \mathscr{H}_{V}^{q+1}(\mathscr{F})\right)$ are coherent on $X$.

(ii) For every $x \in V, \theta_{0}(\mathscr{F} \mid X-V)_{x}, \ldots, \theta_{q}(\mathscr{F} \mid X-V)_{x}$ (or equivalently $\mathscr{H}_{V}^{0}(\mathscr{F})_{x}$, $\left.\ldots, \mathscr{H}_{V}^{q+1}(\mathscr{F})_{x}\right)$ are finitely generated over $\mathscr{H}_{x}$.

(iii) $\operatorname{dim} V \cap \bar{S}_{k+q+1}(\mathscr{F} \mid X-V)<k$ for every $k \geqq 0$.

THEOREM B. Suppose $V$ is an algebraic subvariety of a complex algebraic space $X$, $q$ is a nonnegative integer, and $\mathscr{F}$ is a coherent algebraic sheaf on $X$. Then $\mathscr{H}_{V}^{0}(\mathscr{F})$, $\ldots, \mathscr{H}_{V}^{a+1}(\mathscr{F})$ are coherent algebraic sheaves on $X$ if and only if $\mathscr{H}_{V}^{0}\left(\mathscr{F}^{n}\right), \ldots$, $\mathscr{H}_{V}^{q+1}\left(\mathscr{F}^{h}\right)$ are coherent analytic sheaves on $X$. If so, the canonical homomorphisms $\mathscr{H}_{V}^{k}(\mathscr{F})^{h} \rightarrow \mathscr{H}_{v}^{k}\left(\mathscr{F}^{h}\right)$ are isomorphisms for $0 \leqq k \leqq q+1$.

These results are announced without proofs in [15].

Complex analytic spaces, in this paper, are in the sense of Grauert (i.e., not necessarily reduced). A holomorphic function $f$ on a complex analytic space $(X, \mathscr{H})$ means an element of $\Gamma(X, \mathscr{H})$. We say that $f$ vanishes at a point $x$ of $X$ if $f_{x}$ is not a unit in $\mathscr{H}_{x}$.

If $n$ is a nonnegative integer and $\mathscr{F}$ is a coherent analytic sheaf on a complex analytic space $X$, then $O_{[n]}$ denotes the subsheaf of $\mathscr{F}$ defined as follows: for $x \in X,\left(O_{[n] \mathscr{F}}\right)_{x}=\left\{s \in \mathscr{F}_{x} \mid\right.$ for some open neighborhood $U$ of $x$ in $X, s$ is induced by some $t \in \Gamma(U, \mathscr{F})$ satisfying $\operatorname{dim} \operatorname{Supp} t \leqq n\} . O_{[n] \mathscr{F}}$ is coherent and $\operatorname{dim} \operatorname{Supp} O_{[n]}$ $\leqq n$ (see [10] and [16]). If $V$ is an analytic subvariety of $X$ and $\mathscr{G}$ is a coherent analytic subsheaf of $\mathscr{F}$, then $\mathscr{G}[V]_{\mathscr{F}}$ denotes the subsheaf of $\mathscr{F}$ defined as follows: $\left(\mathscr{G}[V]_{\mathscr{F}}\right)_{x}=\left\{s \in \mathscr{F}_{x} \mid\right.$ for some open neighborhood $U$ of $x$ in $X, s$ is induced by some $t \in \Gamma(U, \mathscr{F})$ such that $t_{y} \in \mathscr{G}_{y}$ for $\left.y \in U-V\right\}$. $\mathscr{G}[V]_{\mathscr{F}}$ is coherent and $\operatorname{Supp}\left(\mathscr{G}[V]_{\mathscr{F}} / \mathscr{G}\right) \subset V$ (see [10] and [16]).

If $\mathscr{G}$ is an analytic subsheaf of an analytic sheaf $\mathscr{F}$ on a complex analytic space $(X, \mathscr{H})$ and $\mathscr{I}$ is an analytic ideal sheaf on $X$, then $(\mathscr{G}: \mathscr{I})_{\mathscr{F}}$ denotes the largest analytic subsheaf $\mathscr{S}$ of $\mathscr{F}$ such that $\mathscr{I} \mathscr{S} \subset \mathscr{G}$.

The same notations are also used for the complex algebraic case. When a space has both analytic and algebraic structures, the symbols $\mathcal{O}$ and $\mathscr{H}$ denote respectively the algebraic and analytic structure-sheaves.

All rings are commutative and have identity. Subrings share the same identity elements as the rings they are imbedded in. All modules are unitary. If $R$ is a ring and $N \subset M$ are $R$-modules and $I$ is an ideal in $R$, then $(N: I)_{M}$ is the largest $R$ submodule $S$ of $M$ such that $I S \subset N$. If $I=R f$ for some $f \in R$, then $(N: I)_{M}$ is also denoted by $(N: f)_{M}$. 
LEMMA 1. Suppose $G$ is an open subset of $C^{n}$ and $V, Y, Z$ are analytic subvarieties of $G$. Let $\sigma: G-Y \rightarrow G$ be the inclusion map. Suppose $\mathscr{F}$ and $\mathscr{G}$ are coherent analytic sheaves on $G$ such that $O[Y]_{\mathscr{F}}=O[Y]_{\mathscr{G}}=0$. Suppose $\varphi: \mathscr{F} \rightarrow \mathscr{G}$ is a sheafhomomorphism on $G$ such that $\varphi$ is an isomorphism on $G-(V \cup Z)$. Suppose $p, q$ are nonnegative integers, $f$ is a holomorphic function on $G$ vanishing identically on $V$, and $Q$ is a relatively compact open subset of $G$ such that $f^{p} \sigma_{k}(\mathscr{G})=0$ on $Q-Z$ for $1 \leqq k \leqq q$ and $f^{p} \sigma_{0}(\mathscr{G}) \subset \mathscr{G}$ on $Q-Z$. Then there exists $p^{\prime} \geqq 0$ such that $f^{p^{\prime}} \sigma_{k}(\mathscr{F})=0$ on $Q-Z$ for $1 \leqq k \leqq q$ and $f^{p^{\prime}} \sigma_{0}(\mathscr{F}) \subset \mathscr{F}$ on $Q-Z$.

Before we prove Lemma 1, we have to clarify one point in its statement. There are canonical maps $\xi: \mathscr{F} \rightarrow \sigma_{0}(\mathscr{F})$ and $\eta: \dot{G} \rightarrow \sigma_{0}(\mathscr{G})$. It is obvious that Ker $\xi$ $=O[Y]_{\mathscr{F}}$ and $\operatorname{Ker} \eta=O[Y]_{\mathscr{G}}$. Since $O[Y]_{\mathscr{F}}=O[Y]_{\mathscr{G}}=0$, we can identify $\mathscr{F}$ and $\mathscr{G}$ as subsheaves of $\sigma_{0}(\mathscr{F})$ and $\sigma_{0}(\mathscr{G})$ through $\xi$ and $\eta$ respectively. $f^{p} \sigma_{0}(\mathscr{G}) \subset \mathscr{G}$ means that, as subsheaves of $\sigma_{0}(\mathscr{G}), f^{p} \sigma_{0}(\mathscr{G})$ is contained in $\mathscr{G} . f^{p^{\prime}} \sigma_{0}(\mathscr{F}) \subset \mathscr{F}$ has a similar meaning. Similar situations arise frequently in this paper and they should be interpreted similarly.

Proof of Lemma 1. Let $\mathscr{K}=\operatorname{Ker} \varphi, \mathscr{R}=\operatorname{Im} \varphi$, and $\mathscr{L}=$ Coker $\varphi$. Supp $\mathscr{K} \subset V \cup Z$ and Supp $\mathscr{L} \subset V \cup Z$. Let $\mathscr{I}=O[V]_{\mathscr{X}}$ and $\mathscr{J}=O[V]_{\mathscr{L}}$. Supp $\mathscr{I} \subset V$ and Supp $\mathscr{J} \subset V$. Since $Q$ is relatively compact in $G$ and $f$ vanishes identically on $V$, there exists $r \geqq 0$ such that $f^{r} \mathscr{I}=f^{r} \mathscr{J}=0$ on $Q$. Since $\mathscr{I}=\mathscr{K}$ on $G-Z$ and $\mathscr{J}=\mathscr{L}$ on $G-Z$, $f^{r} \mathscr{K}=f^{r} \mathscr{L}=0$ on $Q-Z$.

Since $0 \rightarrow \mathscr{R} \rightarrow \mathscr{G} \rightarrow \mathscr{L} \rightarrow 0$ is exact, $\sigma_{k-1}(\mathscr{L}) \rightarrow \sigma_{k}(\mathscr{R}) \rightarrow \sigma_{k}(\mathscr{G})$ is exact for $1 \leqq k \leqq q$. Since $f^{r} \mathscr{L}=0$ on $Q-Z, f^{r} \sigma_{k-1}(\mathscr{L})=0$ on $Q-Z$. Since $f^{p} \sigma_{k}(\mathscr{G})=0$ on $Q-Z$ for $1 \leqq k \leqq q, f^{p+r} \sigma_{k}(\mathscr{R})=0$ on $Q-Z$ for $1 \leqq k \leqq q$.

Since $f^{r} \mathscr{L}=0$ on $Q-Z$ and $f^{p} \sigma_{0}(\mathscr{G}) \subset \mathscr{G}$ on $Q-Z$, from the exact sequence $0 \rightarrow \mathscr{R} \rightarrow \mathscr{G} \rightarrow \mathscr{L}$ we conclude readily that $f^{p+r} \sigma_{0}(\mathscr{R}) \subset \mathscr{R}$ on $Q-Z$.

Since $0 \rightarrow \mathscr{K} \rightarrow \mathscr{F} \rightarrow \mathscr{R} \rightarrow 0$ is exact, $\sigma_{k}(\mathscr{K}) \rightarrow \sigma_{k}(\mathscr{F}) \rightarrow \sigma_{k}(\mathscr{R})$ is exact for $1 \leqq k \leqq q$. Since $f^{r} \mathscr{K}=0$ on $Q-Z, f^{r} \sigma_{k}(\mathscr{K})=0$ on $Q-Z$. Since $f^{p+r} \sigma_{k}(\mathscr{R})=0$ on $Q-Z$ for $1 \leqq k \leqq q, f^{p+2 r} \sigma_{k}(\mathscr{F})=0$ on $Q-Z$ for $1 \leqq k \leqq q$.

Since $f^{r} \sigma_{0}(\mathscr{K})=0$ on $Q-Z$ and $f^{p+r} \sigma_{0}(\mathscr{R}) \subset \mathscr{R}$ on $Q-Z$, from the exact sequence $\mathscr{K} \rightarrow \mathscr{F} \rightarrow \mathscr{R} \rightarrow 0$ we conclude readily that $f^{p+2 r} \sigma_{0}(\mathscr{F}) \subset \mathscr{F}$ on $Q-Z$.

Hence $p^{\prime}=p+2 r$ satisfies the requirement. Q.E.D.

Lemma 2. Suppose $G$ is an open subset of $C^{n}$ and $Y, V, Z$ are analytic subvarieties of $G$ such that $\operatorname{dim}(Y-Z)=r \leqq n-2$ and $Y \subset V$. Let $\sigma: G-Y \rightarrow G$ be the inclusion map. Suppose $\mathscr{F}$ is a coherent analytic sheaf on $G$ such that $O[Y]_{\mathscr{F}}=0$ and $\mathscr{F}$ is locally free on $G-(V \cup Z)$. If $f$ is a holomorphic function on $G$ vanishing identically on $V$ and $Q$ is a relatively compact open subset of $G$, then there exists $s \geqq 0$ such that $f^{s} \sigma_{k}(\mathscr{F})=0$ on $Q-Z$ for $1 \leqq k \leqq n-r-2$ and $f^{s} \sigma_{0}(\mathscr{F}) \subset \mathscr{F}$ on $Q-Z$.

Proof. By replacing $Y$ by $(Y-Z)^{-}$, we can assume w.l.o.g. that $\operatorname{dim} Y=r$.

Let $\mathscr{F}^{*}=\operatorname{Hom}_{\mathscr{H}}\left(\operatorname{Hom}_{\mathscr{H}}(\mathscr{F}, \mathscr{H}), \mathscr{H}\right)$, where $\mathscr{H}=$ the analytic structure-sheaf of $C^{n}$. Since $Q$ is relatively compact in $G$, the problem is local in nature. We can 
assume w.l.o.g. that we have the following exact sequence on $G$ :

$$
\mathscr{H}^{p_{n-r-1}} \rightarrow \mathscr{H}^{p_{n-r-2}} \rightarrow \cdots \rightarrow \mathscr{H}^{p_{1}} \rightarrow \mathscr{H}^{p_{0}} \rightarrow \operatorname{Hom}_{\mathscr{H}}(\mathscr{F}, \mathscr{H}) \rightarrow 0 .
$$

By applying the functor $\operatorname{Hom}_{\mathscr{H}}(\cdot, \mathscr{H})$, we obtain

$$
\begin{aligned}
0 \longrightarrow & \mathscr{F}^{*} \longrightarrow \mathscr{H}^{p_{0}} \stackrel{\varphi_{0}}{\longrightarrow} \mathscr{H}^{p_{1}} \stackrel{\varphi_{1}}{\longrightarrow} \cdots \\
& \stackrel{\varphi_{n-r-3}}{\longrightarrow} \mathscr{H}^{p_{n-r-2}} \stackrel{\varphi_{n-r-2}}{\longrightarrow} \mathscr{H}^{p_{n-r-1}}
\end{aligned}
$$

Since $\mathscr{F}$ is locally free on $G-(V \cup Z), \operatorname{Hom}_{\mathscr{H}}(\mathscr{F}, \mathscr{H})$ is locally free on $G-$ $(V \cup Z)$. (1) is therefore exact on $G-(V \cup Z)$.

Let $\mathscr{G}_{v}=\operatorname{Ker} \varphi_{v}$ and $\mathscr{L}_{v}=\operatorname{Im} \varphi_{v-1}$. Since $r \leqq n-2, \sigma_{0}(\mathscr{H})=\mathscr{H}$. Hence, for $\nu \geqq 0, \sigma_{0}\left(\mathscr{G}_{v}\right)=\mathscr{G}_{v}[Y]_{\mathscr{H}^{p_{v}}}$ and $\sigma_{0}\left(\mathscr{L}_{v+1}\right)=\mathscr{L}_{v+1}[Y]_{\mathscr{H}^{p_{v+1}}}$. Since $\varphi_{v}$ is defined by a matrix of holomorphic functions, $\mathscr{G}_{v}[Y]_{\mathscr{H}^{p_{v}}}=\mathscr{G}_{v}$. Therefore

$$
\sigma_{0}\left(\mathscr{G}_{v}\right)=\mathscr{G}_{v} \text { for } \nu \geqq 0 .
$$

Since $\operatorname{Supp}\left(\mathscr{L}_{v+1}[Y]_{\mathscr{H}^{p_{v+1}}} / \mathscr{L}_{v+1}\right) \subset Y$, there exists a nonnegative integer $\alpha_{v}$ such that

$$
f^{\alpha} \sigma_{0}\left(\mathscr{L}_{v+1}\right) \subset \mathscr{L}_{v+1} \text { on } Q
$$

For $0 \leqq \nu \leqq n-r-2$ consider :

$$
\text { for some nonnegative } s_{v}, f^{s_{v}} \sigma_{k}\left(\mathscr{G}_{v}\right)=0
$$

$$
\text { on } Q-Z \text { for } 1 \leqq k \leqq n-r-2-\nu \text {. }
$$

We are going to prove (4) $)_{v}$ by descending induction on $\nu$. (4) $)_{n-r-2}$ is a vacuous statement and hence is true.

For the general case, suppose $0 \leqq \nu<n-r-2$ and $(4)_{v+1}$ is true. Since (1) is exact on $G-(V \cup Z), \mathscr{L}_{v+1}$ agrees with $\mathscr{G}_{v+1}$ on $G-(V \cup Z)$. By (2) and Lemma 1, there exists a nonnegative integer $t_{v}$ such that $f^{t_{v} \sigma_{k}}\left(\mathscr{L}_{v+1}\right)=0$ on $Q-Z$ for $1 \leqq k \leqq n-r-2-(\nu+1)$.

$0 \rightarrow \mathscr{G}_{v} \rightarrow \mathscr{H}^{p_{v}} \rightarrow \mathscr{L}_{v+1} \rightarrow 0$ is exact. For $1<k \leqq n-r-2-\nu, \quad \sigma_{k-1}\left(\mathscr{L}_{v+1}\right)$

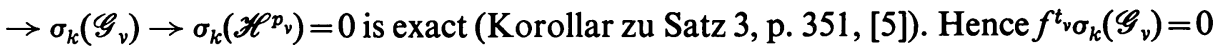
on $Q-Z$ for $1<k \leqq n-r-2-\nu . \sigma_{0}\left(\mathscr{H}^{p_{v}}\right) \rightarrow \sigma_{0}\left(\mathscr{L}_{v+1}\right) \rightarrow \sigma_{1}\left(\mathscr{G}_{v}\right) \rightarrow \sigma_{1}\left(\mathscr{H}^{p_{v}}\right)=0$ is exact (Korollar zu Satz 3, p. 351, [5]). Since $\sigma_{0}\left(\mathscr{H}^{p_{v}}\right)=\mathscr{H}^{p_{v}}, \operatorname{Im}\left(\sigma_{0}\left(\mathscr{H}^{p_{v}}\right) \rightarrow \sigma_{0}\left(\mathscr{L}_{v+1}\right)\right.$ )

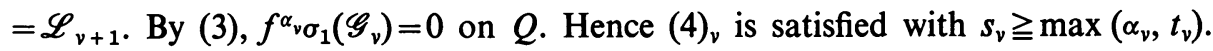
The induction process is complete.

Since (1) is exact on $G-(V \cup Z), \mathscr{F} *$ agrees with $\mathscr{G}_{0}$ on $G-(V \cup Z)$. By (2), (4) $)_{0}$, and Lemma 1 , for some nonnegative integer $s^{\prime}, f^{s^{\prime}} \sigma_{0}\left(\mathscr{F}^{*}\right) \subset \mathscr{F}^{*}$ on $Q-Z$ and $f^{s^{\prime}} \sigma_{k}\left(\mathscr{F}^{*}\right)=0$ on $Q-Z$ for $1 \leqq k \leqq n-r-2$.

Since $\mathscr{F}$ is locally free on $G-(V \cup Z)$, the natural map $\mathscr{F} \rightarrow \mathscr{F} *$ is isomorphic on $G-(V \cup Z)$. By Lemma 1 , there exists a nonnegative integer $s$ such that $f^{s} \sigma_{0}(\mathscr{F}) \subset \mathscr{F}$ on $Q-Z$ and $f^{s} \sigma_{k}(\mathscr{F})=0$ on $Q-Z$ for $1 \leqq k \leqq n-r-2$. Q.E.D. 
Proposition 1. Suppose $G$ is an open subset of $C^{n}$ and $Y, V, Z$ are analytic subvarieties of $G$ such that $Y \subset V$. Let $\sigma: G-Y \rightarrow G$ be the inclusion map. Suppose $l$ is a nonnegative integer and $\mathscr{F}$ is a coherent analytic sheaf on $G$ such that $O[Y]_{\mathscr{F}}=0$ and $\operatorname{codh} \mathscr{F} \geqq \operatorname{dim}(Y-Z)+l+2$ on $G-(V \cup Z)$. If $f$ is a holomorphic function on $G$ vanishing identically on $V$ and $Q$ is a relatively compact open subset of $G$, then there exists a nonnegative integer $s$ such that $f^{s} \sigma_{k}(\mathscr{F})=0$ on $Q-Z$ for $1 \leqq k \leqq l$ and $f^{s} \sigma_{0}(\mathscr{F}) \subset \mathscr{F}$ on $Q-Z$.

Proof. We can assume w.l.o.g. that $\mathscr{F} \neq 0$. Let $r=\operatorname{dim}(Y-Z)$. Then $l \leqq n-r-2$. We are going to prove the proposition by descending induction on $l$.

When $l=n-r-2, \mathscr{F}$ is locally free on $G-(V \cup Z)$ and the proposition follows from Lemma 2.

For the general case, suppose $l<n-r-2$. Since $Q$ is relatively compact, the problem is local in nature. We can assume w.l.o.g. that we have an exact sequence $0 \rightarrow \mathscr{G} \rightarrow \mathscr{H}^{p} \rightarrow \mathscr{F} \rightarrow 0$ on $G$, where $\mathscr{H}$ is the analytic structure-sheaf of $C^{n}$. $\operatorname{codh} \mathscr{G} \geqq r+l+3=r+(l+1)+2$ on $G-(V \cup Z)$.

By induction hypothesis, for some nonnegative integer $s, f^{s} \sigma_{k}(\mathscr{G})=0$ on $Q-Z$ for $1 \leqq k \leqq l+1$ and $f^{s} \sigma_{0}(\mathscr{G}) \subset \mathscr{G}$ on $Q-Z$. For $1 \leqq k \leqq l, 0=\sigma_{k}\left(\mathscr{H}^{p}\right) \rightarrow \sigma_{k}(\mathscr{F})$ $\rightarrow \sigma_{k+1}(\mathscr{G})$ is exact. Hence $f^{s} \sigma_{k}(\mathscr{F})=0$ on $Q-Z$ for $1 \leqq k \leqq l$. $\mathscr{H}^{p}=\sigma_{0}\left(\mathscr{H}^{p}\right) \rightarrow$ $\sigma_{0}(\mathscr{F}) \rightarrow \sigma_{1}(\mathscr{G})$ is exact. $f^{s} \sigma_{0}(\mathscr{F}) \subset \mathscr{F}$ on $Q-Z$. Q.E.D.

Proposition 2. Suppose $V$ is an analytic subvariety of a complex analytic space $X$ and $\mathscr{F}$ is a coherent analytic sheaf on $X$ such that $O[V]_{\mathscr{F}}=0$. Let $\theta: X-V \rightarrow X$ be the inclusion map. Suppose $q$ is a nonnegative integer and $f$ is a holomorphic function on $X$ such that $f \theta_{0}(\mathscr{F}) \subset \mathscr{F}$ and $f \theta_{k}(\mathscr{F})=0$ for $1 \leqq k \leqq q$. Then $f^{q+1} H^{q}(X-V, \mathscr{F}) \subset \operatorname{Im}\left(H^{q}(X, \mathscr{F}) \rightarrow H^{q}(X-V, \mathscr{F})\right)$.

Proof. Since $f \theta_{0}(\mathscr{F}) \subset \mathscr{F}$, the case $q=0$ is trivial. Hence we assume w.l.o.g. that $q \geqq 1$.

Let

$$
0 \longrightarrow \mathscr{F} \longrightarrow \mathscr{S}_{0} \stackrel{\varphi_{0}}{\longrightarrow} \mathscr{S}_{1} \stackrel{\varphi_{1}}{\longrightarrow} \mathscr{S}_{2} \stackrel{\varphi_{2}}{\longrightarrow} \ldots
$$

be a flabby sheaf resolution on $\mathscr{F}$ on $X$. Applying the functor $\theta_{0}(\cdot)$, we obtain

$$
0 \longrightarrow \mathscr{F}^{*} \longrightarrow \mathscr{S}_{0}^{*} \stackrel{\varphi_{0}^{*}}{\longrightarrow} \mathscr{S}_{1}^{*} \stackrel{\varphi_{1}^{*}}{\longrightarrow} \mathscr{S}_{2}^{*} \stackrel{\varphi_{2}^{*}}{\longrightarrow} \cdots
$$

This new sequence is in general not exact. Let $\mathscr{K}_{v}=\operatorname{Ker} \varphi_{v}^{*}$ for $\nu \geqq 0$ and $\mathscr{R}_{v}=$ $\operatorname{Im} \varphi_{v-1}^{*}$ for $\nu \geqq 1$. Then $\mathscr{F}^{*} \approx \operatorname{Ker} \varphi_{0}^{*}$ and $\theta_{v}(\mathscr{F}) \approx \mathscr{K}_{v} / \mathscr{R}_{v}$ for $\nu \geqq 1$.

Let $\mathscr{G}_{v}=\operatorname{Ker} \varphi_{v}$. The natural sheaf-homomorphism $\mathscr{S}_{v} \rightarrow \mathscr{S}_{v}^{*}$ induces natural sheaf-homomorphisms $\mathscr{G}_{v} \rightarrow \mathscr{K}_{v}(\nu \geqq 0)$ and $\mathscr{G}_{v} \rightarrow \mathscr{R}_{v}(v \geqq 1)$.

The following commutative diagram with exact rows

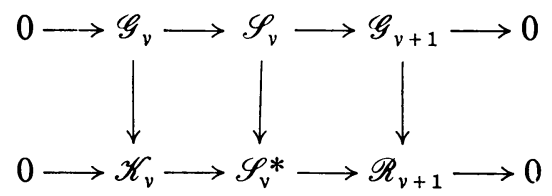


gives rise to the following commutative diagram with exact rows

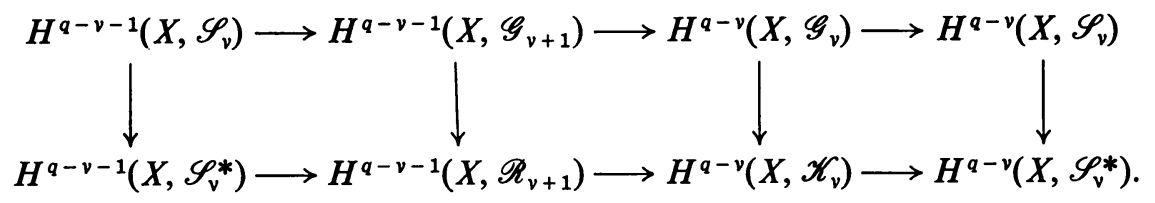

Since $\mathscr{S}_{v}$ and $\mathscr{S}_{v}^{*}$ are both flabby, $H^{q-v-1}\left(X, \mathscr{S}_{v}\right)=H^{q-v}\left(X, \mathscr{S}_{v}\right)=H^{q-v-1}\left(X, \mathscr{S}_{v}^{*}\right)$ $=H^{q-v}\left(X, \mathscr{S}_{v}^{*}\right)=0$ for $0 \leqq \nu<q-1$. Hence

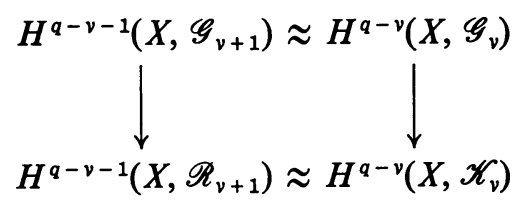

is a commutative diagram for $0 \leqq \nu<q-1$.

We are going to prove $(6)_{v}$ for $0 \leqq \nu \leqq q-1$ by induction on $\nu$ :

$$
f^{v+1} H^{q-v}\left(X, \mathscr{K}_{v}\right) \subset \operatorname{Im}\left(H^{q-v}\left(X, \mathscr{G}_{v}\right) \rightarrow H^{q-v}\left(X, \mathscr{K}_{v}\right)\right) .
$$

The exact sequence $0 \rightarrow \mathscr{F} \rightarrow \mathscr{F} * \rightarrow \mathscr{F}^{*} / \mathscr{F} \rightarrow 0$ gives rise to the exact sequence $H^{q}(X, \mathscr{F}) \rightarrow H^{q}\left(X, \mathscr{F}^{*}\right) \rightarrow H^{q}\left(X, \mathscr{F}^{*} / \mathscr{F}\right)$. Since $f(\mathscr{F} * / \mathscr{F})=0, f H^{q}(X, \mathscr{F} * / \mathscr{F})=0$. $f H^{q}\left(X, \mathscr{F}^{*}\right) \subset \operatorname{Im}\left(H^{q}(X, \mathscr{F}) \rightarrow H^{q}\left(X, \mathscr{F}^{*}\right)\right)$. (6) $)_{0}$ follows from $\mathscr{G}_{0} \approx \mathscr{F}$ and $\mathscr{K}_{0} \approx \mathscr{F} *$.

For the general case, assume $0<\nu \leqq q-1$ and that $(6)_{v-1}$ is true. By (5) and $(6)_{v-1}$,

$$
f^{v} H^{q-v}\left(X, \mathscr{R}_{v}\right) \subset \operatorname{Im}\left(H^{q-v}\left(X, \mathscr{G}_{v}\right) \rightarrow H^{q-v}\left(X, \mathscr{R}_{v}\right)\right) .
$$

The followimg commutative diagram with exact rows

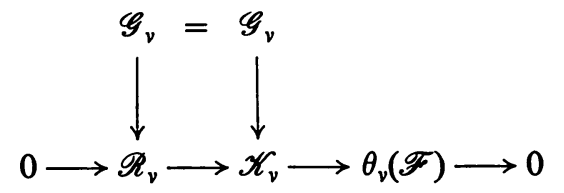

gives rise to the following commutative diagram with exact rows

(8)

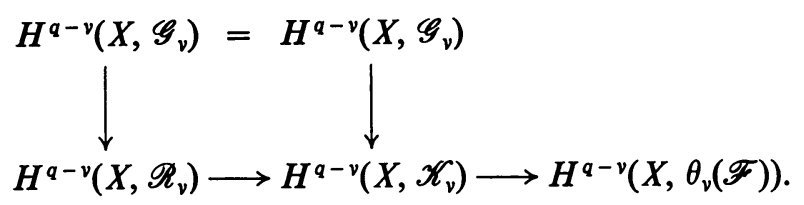

Since $f \theta_{v}(\mathscr{F})=0, f H^{q-v}\left(X, \theta_{v}(\mathscr{F})\right)=0$. Hence $(6)_{v}$ follows from (7) and (8). The induction process is completed.

The exact sequence $0 \rightarrow \mathscr{R}_{q} \rightarrow \mathscr{K}_{q} \rightarrow \theta_{q}(\mathscr{F}) \rightarrow 0$ yields the exact sequence $0 \rightarrow \Gamma\left(X, \mathscr{R}_{q}\right) \rightarrow \Gamma\left(X, \mathscr{K}_{q}\right) \rightarrow \Gamma\left(X, \theta_{q}(\mathscr{F})\right)$. We identify $\Gamma\left(X, \mathscr{R}_{q}\right)$ as a subset of $\Gamma\left(X, \mathscr{K}_{q}\right)$. Since $f \theta_{q}(\mathscr{F})=0, f \Gamma\left(X, \theta_{q}(\mathscr{F})\right)=0$.

$$
f \Gamma\left(X, \mathscr{K}_{q}\right) \subset \Gamma\left(X, \mathscr{R}_{q}\right) .
$$


The following commutative diagram with exact rows

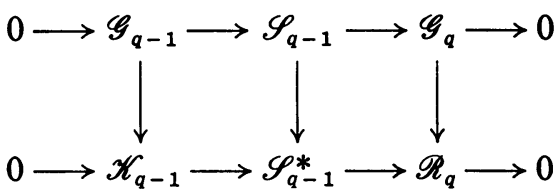

yields the following commutative diagram with exact rows

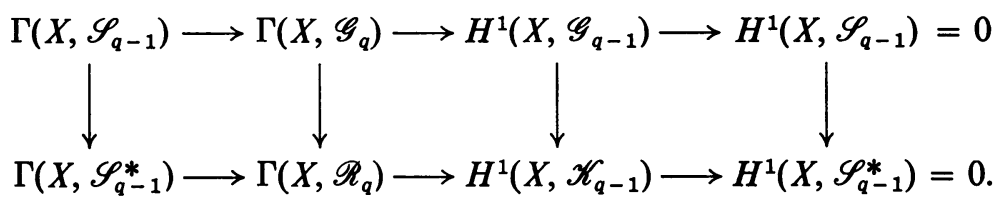

Since $\operatorname{Im}\left(\Gamma\left(X, \mathscr{S}_{q-1}\right) \rightarrow \Gamma\left(X, \mathscr{G}_{q}\right)\right)=\operatorname{Im}\left(\Gamma\left(X, \mathscr{S}_{q-1}\right) \rightarrow \Gamma\left(X, \mathscr{S}_{q}\right)\right)$ and

$$
\operatorname{Im}\left(\Gamma\left(X, \mathscr{S}_{q-1}^{*}\right) \rightarrow \Gamma\left(X, \mathscr{R}_{q}\right)\right)=\operatorname{Im}\left(\Gamma\left(X, \mathscr{S}_{q-1}^{*}\right) \rightarrow \Gamma\left(X, \mathscr{S}_{q}^{*}\right)\right),
$$

the following diagram is commutative:

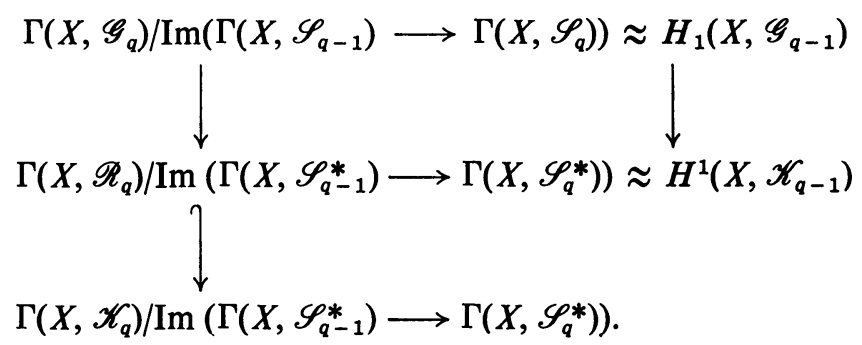

On the other hand, we have

$$
\begin{aligned}
H^{q}(X, \mathscr{F}) & \approx \Gamma\left(X, \mathscr{G}_{q}\right) / \operatorname{Im}\left(\Gamma\left(X, \mathscr{S}_{q-1}\right) \rightarrow \Gamma\left(X, \mathscr{S}_{q}\right)\right) \\
H^{q}(X-V, \mathscr{F}) & \approx \Gamma\left(X, \mathscr{K}_{q}\right) / \operatorname{Im}\left(\Gamma\left(X, \mathscr{S}_{q-1}^{*}\right) \rightarrow \Gamma\left(X, \mathscr{S}_{q}^{*}\right)\right),
\end{aligned}
$$

because

and

$$
\begin{aligned}
H^{q}(X, \mathscr{F}) & \approx \operatorname{Ker}\left(\Gamma\left(X, \mathscr{S}_{q}\right) \rightarrow \Gamma\left(X, \mathscr{S}_{q+1}\right)\right) / \operatorname{Im}\left(\Gamma\left(X, \mathscr{S}_{q-1}\right) \rightarrow \Gamma\left(X, \mathscr{S}_{q}\right)\right) \\
& =\Gamma\left(X, \mathscr{G}_{q}\right) / \operatorname{Im}\left(\Gamma\left(X, \mathscr{S}_{q-1}\right) \rightarrow \Gamma\left(X, \mathscr{S}_{q}\right)\right)
\end{aligned}
$$

$$
\begin{aligned}
H^{q}(X-V, \mathscr{F}) & \\
& \approx \operatorname{Ker}\left(\Gamma\left(X-V, \mathscr{S}_{q}\right) \rightarrow \Gamma\left(X-V, \mathscr{S}_{q+1}\right)\right) / \operatorname{Im}\left(\Gamma\left(X-V, \mathscr{S}_{q-1}\right) \rightarrow \Gamma\left(X-V, \mathscr{S}_{q}\right)\right) \\
& =\operatorname{Ker}\left(\Gamma\left(X, \mathscr{S}_{q}^{*}\right) \rightarrow \Gamma\left(X, \mathscr{S}_{q+1}^{*}\right)\right) / \operatorname{Im}\left(\Gamma\left(X, \mathscr{S}_{q-1}^{*}\right) \rightarrow \Gamma\left(X, \mathscr{S}_{q}^{*}\right)\right) \\
& =\Gamma\left(X, \mathscr{K}_{q}\right) / \operatorname{Im}\left(\Gamma\left(X, \mathscr{S}_{q-1}^{*}\right) \rightarrow \Gamma\left(X, \mathscr{S}_{q}^{*}\right)\right) .
\end{aligned}
$$

The proposition follows from (6) $)_{q-1},(9),(10)$, and (11). Q.E.D.

Proposition 3. Suppose $q$ is a nonnegative integer, $G$ is an open subset of $C^{n}$, and $Q$ is a relatively compact open subset of $G$. Suppose $V, Z$ are analytic subvarieties of $G$ and $f$ is a holomorphic function on $G$ vanishing identically on $V$. Let $\theta: G-V \rightarrow G$ 
be the inclusion map. Suppose $\mathscr{F}$ is a coherent analytic sheaf on $G$ such that (i) $O[V]_{\mathscr{F}}=0$ and (ii) $\operatorname{dim} A_{k} \cap V<k$ for $k \geqq 0$, where $A_{k}=\bar{S}_{k+q+1}(\mathscr{F} \mid G-V)-Z$. Then there exists a nonnegative integer s such that $f^{s} \theta_{k}(\mathscr{F})=0$ on $Q-Z$ for $1 \leqq k \leqq q$ and $f^{s} \theta_{0}(\mathscr{F}) \subset \mathscr{F}$ on $Q-Z$.

Proof. Let $r$ be the largest integer such that codh $\mathscr{F}>r+q+1$ on $G-(V \cup Z)$. We prove by descending induction on $r$. When $r \geqq n-q-1, \mathscr{F}=0$ on $G-(V \cup Z)$ and the proposition is trivial.

For the general case, assume $r\left\langle n-q-1\right.$. Let $W=A_{r+1}^{-} \cup Z$. Then $\operatorname{codh} \mathscr{F}>$ $r+1+q+1$ on $G-(V \cup W)$. By induction hypothesis, there exists a natural number $s_{1}$ such that $f^{s_{1}} \theta_{k}(\mathscr{F})=0$ on $Q-W$ for $1 \leqq k \leqq q$ and $f^{s_{1}} \theta_{0}(\mathscr{F}) \subset \mathscr{F}$ on $Q-W$.

Let $Y=V \cap W$. Since $\theta_{0}(\mathscr{F})=\mathscr{F}$ on $G-V$ and $\theta_{k}(\mathscr{F})=0$ on $G-V$ for $k \geqq 1$,

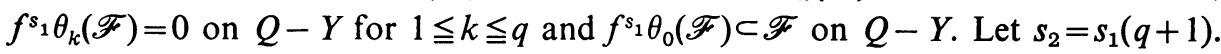
By Proposition 2, for $0 \leqq k \leqq q$ and for any open subset $U$ of $Q, f^{s_{2}} H^{k}(U-V, \mathscr{F}) \subset$ $\operatorname{Im}\left(H^{k}(U-Y, \mathscr{F}) \rightarrow H^{k}(U-V, \mathscr{F})\right)$. Let $\sigma: G-Y \rightarrow G$ be the inclusion map. Then

$$
f^{s_{2}} \theta_{k}(\mathscr{F}) \subset \operatorname{Im}\left(\sigma_{k}(\mathscr{F}) \rightarrow \theta_{k}(\mathscr{F})\right) \text { for } 0 \leqq k \leqq q .
$$

Since $\operatorname{dim}(Y-Z) \leqq r$ and $\operatorname{codh} \mathscr{F} \geqq q+r+2$ on $G-(V \cup Z)$, by Proposition 1 , there exists a nonnegative integer $s_{3}$ such that

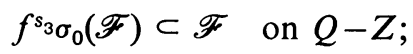

$$
\begin{aligned}
& f^{s_{3} \sigma_{k}(\mathscr{F})=0 \text { on } Q-Z \text { for } 1 \leqq k \leqq q .}
\end{aligned}
$$

Let $s=s_{2}+s_{3}$. The proposition follows from (12) and (13). Q.E.D.

LEMMA 3. Suppose $\mathscr{F}$ is a coherent analytic sheaf on a complex analytic space $(X, \mathscr{H})$. Let $A_{k}$ be the $k$-dimensional component of Supp $O_{[k] \mathscr{F}}$. If $x \in X$ and $f$ is an element of $\mathscr{H}_{x}$ not vanishing identically on any nonempty branch-germ of $A_{k}$ at $x$ for any $k \geqq 0$, then $f$ is not a zero-divisor for $\mathscr{F}_{x}$.

Proof. Suppose the contrary. Then we can find an open neighborhood $U$ of $x$ in $X, g \in \Gamma(U, \mathscr{H})$, and $h \in \Gamma(U, \mathscr{F})$ such that $g_{x}=f, h_{x} \neq 0$, and $g h=0$. Let $Z=\operatorname{Supp} h$ and $k=\operatorname{dim}_{x} Z$. Then $k \geqq 0$. By shrinking $U$, we can assume that $\operatorname{dim} Z=k . \quad h \in \Gamma\left(U, O_{[k] \mathscr{F}}\right) . \quad Z \subset \operatorname{Supp} O_{[k] \mathscr{F}}$. Since $\operatorname{dim} \operatorname{Supp} O_{[k] \mathscr{F}} \leqq k$, any $k-$ dimensional branch-germ of $Z$ at $x$ is a $k$-dimensional branch-germ of Supp $O_{[k] \mathscr{F} \text {. }}$ $g_{x}$ vanishes identically on a nonempty branch-germ of $A_{k}$ at $x$, because $g h=0$. Contradiction. Q.E.D.

LEMma 4. Suppose $\mathscr{F}$ is a coherent analytic sheaf on a complex analytic space $(X, \mathscr{H})$. Then, for any $k \geqq 0$, the $k$-dimensional component of Supp $O_{[k]}$ equals the $k$-dimensional component of $S_{k}(\mathscr{F})$.

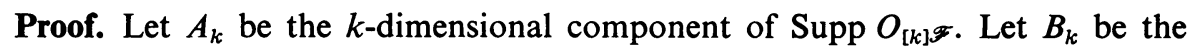
$k$-dimensional component of $S_{k}(\mathscr{F})$. 
Fix $k \geqq 0$. By Satz I, p. 359 of [5], $O_{[k] \mathscr{F}}=0$ on $X-S_{k}(\mathscr{F})$. Hence $A_{k} \subset B_{k}$. To prove $B_{k} \subset A_{k}$, assume the contrary. Then $B_{k} \notin \bigcup_{l=0}^{k} A_{l}$. Take $x \in B_{k}-\bigcup_{l=0}^{k} A_{l}$. Choose an open neighborhood $U$ of $x$ in $X-\bigcup_{l=0}^{k} A_{l}$ and $f \in \Gamma(U, \mathscr{H})$ such that (i) $f$ vanishes identically on $B_{k} \cap U$ and (ii) for every $l>k$ and every $y \in U, f_{y}$ does not vanish identically on any nonempty branch-germ of $A_{l}$ at $y$. This is possible, because $\operatorname{dim} A_{l}>\operatorname{dim} B_{k}$ for $l>k$. By Lemma $3, f_{y}$ is not a zero-divisor for $\mathscr{F}_{y}$ for $y \in U$. We have codh $\mathscr{F}_{x} \geqq 1$. This is a contradiction if $k=0$. If $k>0$, then $\operatorname{dim} S_{k-1}((\mathscr{F} \mid f \mathscr{F}) \mid U) \leqq k-1[6$, Satz 5, p. 81]). There exists

$$
z \in B_{k} \cap U-S_{k-1}((\mathscr{F} \mid f \mathscr{F}) \mid U) .
$$

$\operatorname{codh}(\mathscr{F} \mid f \mathscr{F})_{z}>k-1$. codh $\mathscr{F}_{z}>k$, contradicting $z \in B_{k}$. Q.E.D.

LEMMA 5. If $Y$ is a nowhere dense ( $n-1)$-dimensional analytic subvariety of an $n$-dimensional complex analytic space $X$, then there exists a point $x$ of $Y$ and an open neighborhood $U$ of $x$ in $X$ such that $U-Y$ is Stein.

Proof. Clearly we can assume that $X$ is reduced. Let $X^{\prime}$ be the union of all branches of $X$ which have dimension $<n$ and let $Y^{\prime}$ be the union of all branches of $Y$ which have dimension $<n-1$. By replacing $X$ by $X-X^{\prime}-Y^{\prime}$ and $Y$ by $Y-X^{\prime}-Y^{\prime}$, we can assume that both $X$ and $Y$ are of pure dimension. Let $S$ be the set of all singular points of $X$.

Case 1. $Y \notin S$. Let $T$ be the set of all singular points of $Y$. By replacing $X$ by $X-S-T$ and $Y$ by $Y-S-T$, we can assume that both $X$ and $Y$ are regular. Take $x \in Y$. There exists a Stein open neighborhood $U$ of $x$ in $X$ such that $U \cap Y$ is the zero-set of a single holomorphic function on $U$. $U-Y$ is Stein.

Case 2. $Y \subset S$. Take an $(n-1)$-dimensional branch $Y_{0}$ of $Y$. Since $\operatorname{dim} S$ $\leqq n-1, Y_{0}$ is a branch of $S$. Let $Y_{1}$ be the union of all branches of $Y$ other than $Y_{0}$ and let $S_{1}$ be the union of all branches of $S$ other than $Y_{0}$. By replacing $X$ by $X-Y_{1}-S_{1}$ and $Y$ by $Y-Y_{1}-S_{1}$, we can assume that $Y=S$. Let $\pi: \tilde{X} \rightarrow X$ be the normalization of $X$. Let $\tilde{Y}=\pi^{-1}(Y)$. Let $\tilde{T}$ be the set of all singular points of $\tilde{Y}$ and let $\tilde{S}$ be the set of all singular points of $\tilde{X} . \operatorname{dim} \tilde{T} \leqq n-2$ and $\operatorname{dim} \tilde{S} \leqq n-2$. Take $x \in Y-\pi(\tilde{T} \cup \tilde{S})$. Let $\pi^{-1}(x)=\left\{x_{1}, \ldots, x_{k}\right\}$. For $1 \leqq i \leqq k$, there exists a Stein open neighborhood $U_{i}$ of $x_{i}$ in $\tilde{X}$ such that (i) $\tilde{Y} \cap U_{i}$ is the zero-set of a single holomorphic function on $U_{i}$ and (ii) $U_{i} \cap U_{j}=\varnothing$ for $i \neq j$. Choose a Stein open neighborhood $U$ of $x$ in $X$ such that $\pi^{-1}(U) \subset \bigcup_{i=1}^{k} U_{i} \cdot \pi^{-1}(U)-\tilde{Y}$ is Stein. Since $U-Y$ is biholomorphic to $\pi^{-1}(U)-\tilde{Y}, U-Y$ is Stein. Q.E.D.

Lemma 6. Suppose $Y$ is a nowhere dense (n-1)-dimensional analytic subvariety of an n-dimensional complex analytic space $(X, \mathscr{H})$. Let $\sigma: X-Y \rightarrow X$ be the inclusion map. Suppose $\mathscr{F}$ is a coherent analytic sheaf on $X$ such that $\operatorname{Supp} \mathscr{F}=X$. Then for some $X \in Y, \sigma_{0}(\mathscr{F})_{x}$ is not finitely generated over $\mathscr{H}_{x}$.

Proof. By Lemma 5, we can choose a point $x$ of $Y$ and an open neighborhood $U$ of $x$ in $X$ such that $U-Y$ is Stein. We are going to prove that $\sigma_{0}(\mathscr{F})_{x}$ is not finitely generated over $\mathscr{H}_{x}$. Suppose the contrary. 
Choose a sequence $\left\{x_{k}\right\}_{k=1}^{\infty}$ in $U-Y$ whose limit is $x$. As a set, $\left\{x_{k}\right\}_{k=1}^{\infty}$ is an analytic subvariety of $U-Y$. Let $\mathscr{I}$ be its analytic ideal-sheaf on $U-Y$.

$$
\Gamma(U-Y, \mathscr{F} / \mathscr{I} \mathscr{F}) \approx \prod_{k=1}^{\infty}(\mathscr{F} / \mathscr{I} \mathscr{F})_{x_{k}} \text {. }
$$

Since $\mathscr{F}_{x_{k}} \neq \varnothing$, by Krull-Azumaya Lemma [4, (4.1)], $(\mathscr{F} / \mathscr{I} \mathscr{F})_{x_{k}} \neq 0$. Choose $s_{k} \in \Gamma(U-Y, \mathscr{F} / \mathscr{I} \mathscr{F})$ such that $\left(s_{k}\right)_{x_{l}}=0$ for $l>k$ and $\left(s_{k}\right)_{x_{k}} \neq 0$.

Since $U-Y$ is Stein, the quotient map $\varphi: \Gamma(U-Y, \mathscr{F}) \rightarrow \Gamma(U-Y, \mathscr{F} / \mathscr{I} \mathscr{F})$ is surjective. For some $u_{k} \in \Gamma(U-Y, \mathscr{F}), \varphi\left(u_{k}\right)=s_{k}$. $u_{k}$ induces an element $v_{k}$ in $\sigma_{0}(\mathscr{F})_{x}$.

Let $M_{k}$ be the $\mathscr{H}_{x}$-submodule of $\sigma_{0}(\mathscr{F})_{x}$ generated by $v_{1}, \ldots, v_{k}$. Since $\sigma_{0}(\mathscr{F})_{x}$ is finitely generated over the Noetherian ring $\mathscr{H}_{x}, M_{k}=M_{k+1}$ for some $k \geqq 1$. Hence, for some neighborhood $D$ of $x$ in $U$, there exist $f_{1}, \ldots, f_{k} \in \Gamma(D, \mathscr{H})$ such that $u_{k+1}=\sum_{i=1}^{k} f_{i} u_{i}$ on $D-Y . s_{k+1}=\sum_{i=1}^{k} f_{i} s_{i}$ on $D-Y .\left(s_{k+1}\right)_{x_{k+1}}=0$. Contradiction. Q.E.D.

Proof of Theorem A. It is obvious that (i) $\Rightarrow$ (ii). To prove (ii) $\Rightarrow$ (iii) and (iii) $\Rightarrow$ (ii), we can assume w.l.o.g. that $X$ is a Stein open subset of $C^{n}$ and $O[V]_{\mathscr{F}}=0$.

First we prove the following:

$$
\text { for } k \geqq 0, V \cap \operatorname{Supp} O_{[k]} \text { is nowhere dense in Supp } O_{[k] \mathscr{F}} \text {. }
$$

Suppose the contrary. Then, for some $k \geqq 0$, we can find an open subset $U$ of $X$ such that $U \cap V \supset U \cap \operatorname{Supp} O_{[k] \mathscr{F}} \neq \varnothing$. On $U, O_{[k] \mathscr{F}} \subset O[V]_{\mathscr{F}}=0$, contradicting $U \cap \operatorname{Supp} O_{[k] \mathscr{F}} \neq \varnothing$.

(a) (ii) $\Rightarrow$ (iii). We prove by induction on $q$. For the case $q=0$, suppose $\operatorname{dim} V$ $\cap \bar{S}_{k+1}(\mathscr{F} \mid X-V) \geqq k$ for some $k \geqq 0$. Let $A$ denote the $(k+1)$-dimensional component of $S_{k+1}(\mathscr{F} \mid X-V)$ and $B$ denote the union of all branches of $S_{k+1}(\mathscr{F} \mid X-V)$ which have dimension $\leqq k$. Since $S_{k+1}(\mathscr{F} \mid X-V)=A \cup B$ and $\operatorname{dim} V \cap B^{-}<k$, we have $A \neq \varnothing$ and $\operatorname{dim} V \cap A^{-}=k$.

Let $Z=\operatorname{Supp} O_{[k+1] \mathscr{F}}$ and $Y=V \cap Z$. By Lemma $4, A=$ the $(k+1)$-dimensional component of $Z-V$. Hence $\operatorname{dim} Y \geqq k$. Since $\operatorname{dim} Z \leqq k+1$ and by (14) $Y$ is nowhere dense in $Z$, we have $\operatorname{dim} Y=k$ and $\operatorname{dim} Z=k+1$.

Let $\mathscr{I}=\left(O: O_{[k+1] \mathscr{F}}\right)_{\mathscr{H}}$. Then $(Z, \mathscr{H} / \mathscr{I})$ is a complex analytic space of dimension $k+1$ and $O_{[k+1] \mathscr{F}}$ can be considered naturally as a coherent analytic sheaf on $(Z, \mathscr{H} \mid \mathscr{I})$.

Let $\sigma: Z-Y \rightarrow Z$ be the inclusion map. By Lemma 6 there exists $x \in Y$ such that $\sigma_{0}\left(O_{[k+1] \mathscr{F}}\right)_{x}$ is not finitely generated over $(\mathscr{H} / \mathscr{I})_{x}$. Since $\sigma_{0}\left(O_{[k+1] \mathscr{F}}\right)_{x}=\theta_{0}\left(O_{[k+1] \mathscr{F}}\right)_{x}$ $\subset \theta_{0}(\mathscr{F})_{x}, \theta_{0}(\mathscr{F})_{x}$ is not finitely generated over $\mathscr{H}_{x}$. Contradiction. The case $q=0$ is proved.

For the general case, assume $q>0$. Suppose (iii) does not hold. Let $k$ be a nonnegative integer such that $\operatorname{dim} V \cap \bar{S}_{k+q+1}(\mathscr{F} \mid X-V) \geqq k$. By induction hypothesis, $\operatorname{dim} V \cap \bar{S}_{l+q+1}(\mathscr{F} \mid X-V) \leqq l$ for every $l \geqq 0$. Hence there exists $x \in V \cap$ $\bar{S}_{k+q+1}(\mathscr{F} \mid X-V)$ such that $x \notin \bar{S}_{k+q}(\mathscr{F} \mid X-V)$.

Choose a Stein open neighborhood $D$ of $x$ in $X$ and an irreducible $(k+1)$ - 
dimensional analytic subvariety $W$ of $\bar{S}_{k+a+1}(\mathscr{F} \mid X-V) \cap D$ such that (i) $D \cap$ $\bar{S}_{k+q}(\mathscr{F} \mid X-V)=\varnothing$ and (ii) $\operatorname{dim} V \cap W=k$. Choose a holomorphic function $f$ on $D$ such that (i) $f$ vanishes identically on $W$ and (ii) $f$ does not vanish identically on any $(l+q+1)$-dimensional branch of $\bar{S}_{l+q+1}(\mathscr{F} \mid X-V) \cap D$ for $l \geqq k$. Since $q \geqq 1$, the existence of such an $f$ follows from dimension arguments.

By Lemmas 3 and $4, f_{y}$ is not a zero-divisor for $\mathscr{F}_{y}$ for $y \in D-V$. On $D$, let $\varphi: \mathscr{F} \rightarrow \mathscr{F}$ be the sheaf-homomorphism defined by multiplication by $f$. Then $\operatorname{Ker} \varphi \subset V \cap D$. Since $O[V]_{\mathscr{F}}=0, \operatorname{Ker} \varphi=0$.

The exact sequence $0 \rightarrow \mathscr{F} \stackrel{\varphi}{\rightarrow} \mathscr{F} \rightarrow \mathscr{F} \mid f \mathscr{F} \rightarrow 0$ on $D$ yields the exact sequence $\theta_{l}(\mathscr{F}) \rightarrow \theta_{l}(\mathscr{F} \mid f \mathscr{F}) \rightarrow \theta_{l+1}(\mathscr{F})$ on $D$ for $l \geqq 0$. Hence $\theta_{0}(\mathscr{F} \mid f \mathscr{F})_{y}, \ldots, \theta_{q-1}(\mathscr{F} \mid f \mathscr{F})_{y}$ are finitely generated over $\mathscr{H}_{y}$ for $y \in V \cap D$.

By induction hypothesis, $\operatorname{dim} V \cap \bar{S}_{l+q}((\mathscr{F} \mid f \mathscr{F}) \mid D-V)<l$ for every $l \geqq 0$. Since $f$ is identically zero on $W$ and $W-V \subset S_{k+q+1}(\mathscr{F} \mid D-V)$,

$$
W-V \subset S_{k+q}((\mathscr{F} \mid f \mathscr{F}) \mid D-V)
$$

and

$\operatorname{dim} V \cap \bar{S}_{k+q}((\mathscr{F} \mid f \mathscr{F}) \mid D-V) \geqq \operatorname{dim} V \cap(W-V)^{-}=\operatorname{dim} V \cap W=k$.

Contradiction. The general case is proved.

(b) (iii) $\Rightarrow$ (i). We prove by induction on $q$. Choose a holomorphic function $f$ on $X$ such that (i) $f$ vanishes identically on $V$ and (ii) $f$ does not vanish identically on any branch of Supp $O_{[k]}$ for any $k \geqq 0$. The choice of such an $f$ is possible because of (14).

Fix arbitrarily $x \in V$. Choose a relatively compact open neighborhood $Q$ of $x$ in $X$.

By Proposition 3, there exists a nonnegative integer $s$ such that $f^{s} \theta_{k}(\mathscr{F})=0$ on $Q$ for $1 \leqq k \leqq q$ and $f^{s} \theta_{0}(\mathscr{F}) \subset \mathscr{F}$ on $Q$. Let $\varphi: \mathscr{F} \rightarrow \mathscr{F}$ and $\tilde{\varphi}: \theta_{0}(\mathscr{F}) \rightarrow \theta_{0}(\mathscr{F})$ be defined by multiplication by $f^{s}$. By Lemma $3, \varphi$ and $\tilde{\varphi}$ are injective.

Since $f^{s} \theta_{0}(\mathscr{F}) \subset \mathscr{F}$ on $Q, \tilde{\varphi}\left(\theta_{0}(\mathscr{F})\right) \subset \mathscr{F}$. On $Q, \tilde{\varphi}\left(\theta_{0}(\mathscr{F})\right)=\varphi(\mathscr{F})[V] \mathscr{F}$ is coherent. Since $\tilde{\varphi}$ is injective, $\theta_{0}(\mathscr{F})$ is coherent on $Q$. Since $x$ is arbitrary, the case $q=0$ is proved.

For the general case, assume $q>0 . S_{k+q}((\mathscr{F} \mid f \mathscr{F}) \mid X-V) \subset S_{k+q+1}(\mathscr{F} \mid X-V)$. Hence $\operatorname{dim} V \cap \bar{S}_{k+q}((\mathscr{F} \mid f \mathscr{F}) \mid X-V)<k$ for every $k \geqq 0$. By induction hypothesis, $\theta_{k}(\mathscr{F})$ and $\theta_{k}(\mathscr{F} \mid f \mathscr{F})$ are coherent on $X$ for $0 \leqq k \leqq q-1$.

The exact sequence $0 \rightarrow \mathscr{F} \stackrel{\varphi}{\rightarrow} \mathscr{F} \rightarrow \mathscr{F} \mid f \mathscr{F} \rightarrow 0$ yields the exact sequence $\theta_{q-1}(\mathscr{F}) \rightarrow \theta_{q-1}(\mathscr{F} \mid f \mathscr{F}) \rightarrow \theta_{q}(\mathscr{F}) \stackrel{\Downarrow}{\rightarrow} \theta_{q}(\mathscr{F})$. Since $f^{s} \theta_{q}(\mathscr{F})=0$ on $Q, \psi=0$ on $Q$. On $Q, \theta_{q}(\mathscr{F})=\operatorname{Coker}\left(\theta_{q-1}(\mathscr{F}) \rightarrow \theta_{q-1}(\mathscr{F} / f \mathscr{F})\right)$ is coherent. Since $x$ is arbitrary, the induction process is complete. Q.E.D.

The complex algebraic analog of Theorem $\mathrm{A}$ is the following:

THeOREM $\mathrm{A}^{\prime}$. Suppose $V$ is an algebraic subvariety of a complex algebraic space $(X, \mathcal{O}), q$ is a nonnegative integer, and $\mathscr{F}$ is a coherent algebraic sheaf on $X$. Let $\theta: X-V \rightarrow X$ be the inclusion map. Then the following three statements are equivalent: 
(i) $\theta_{0}(\mathscr{F} \mid X-V), \ldots, \theta_{q}(\mathscr{F} \mid X-V)\left(\right.$ or equivalently $\left.\mathscr{H}_{V}^{0}(\mathscr{F}), \ldots \mathscr{H}_{V}^{a+1}(\mathscr{F})\right)$ are coherent on $X$.

(ii) For every $x \in V, \theta_{0}(\mathscr{F} \mid X-V)_{x}, \ldots, \theta_{q}(\mathscr{F} \mid X-V)_{x}\left(\right.$ or equivalently $\mathscr{H}_{v}^{0}(\mathscr{F})_{x}$, $\left.\ldots, \mathscr{H}_{V}^{a+1}(\mathscr{F})_{x}\right)$ are finitely generated over $\mathcal{O}_{x}$.

(iii) $\operatorname{dim} V \cap \bar{S}_{k+q+1}(\mathscr{F} \mid X-V)<k$ for every $k \geqq 0$.

The proof of Theorem $\mathrm{A}^{\prime}$ is trivially analogous to the proof of Theorem A. There is no need to repeat the argument. However, since the algebraic analog of Scheja's theorem (Korollar zu Satz 3, p. 351, [5]) on extending cohomology classes which is vital in the proof of Theorem A cannot be proved in the same way as the analytic case, we present what we need for the algebraic case in the following proposition.

Proposition 4. Suppose $V$ is an algebraic subvariety of dimension $r$ in $\boldsymbol{C}^{n}$ and $\mathcal{O}$ is the algebraic structure-sheaf of $\boldsymbol{C}^{n}$. Let $\theta: C^{n}-V \rightarrow C^{n}$ be the inclusion map. Then $\theta_{k}(\mathcal{O})=0$ for $1 \leqq k \leqq n-2-r$ and $\theta_{0}(\mathcal{O})=\mathcal{O}$ if $r \leqq n-2$.

Proposition 4 can be obtained from some general theorems in the works of Grothendieck ([2], [3]). However, for the sake of completeness, we present a direct proof here.

In Lemmas 7 and 8 and the proof of Proposition 4 below, $\Omega$ denotes $C^{n}$ and $\mathcal{O}$ denotes the algebraic structure-sheaf of $C^{n}$. R denotes $\Gamma(\Omega, \mathcal{O})$. If $Q \in R$, then $\Omega_{Q}$ denotes the complement of the zero-set of $Q$ in $\Omega$.

Lemma 7. Suppose $V$ is an algebraic subvariety of $Q, Q \in R$, and $\mathscr{F}$ is a coherent algebraic sheaf on $\Omega_{Q}$. Suppose $p$ is a nonnegative integer and $f \in R$ vanishes identically on $V$. If $s \in H_{V}^{p}\left(\Omega_{Q}, \mathscr{F}\right)$, then there exists a nonnegative integer $m$ (depending on $s$ ), such that $f^{m} s=0$.

Proof. We are going to prove by induction on $p$. Let $V$ be the set of all common zeros of $Q_{1}, \ldots, Q_{k} \in R$.

(a) The case $p=0 . H_{V}^{0}\left(\Omega_{Q}, \mathscr{F}\right)$ is the subset of all elements of $\Gamma\left(\Omega_{Q}, \mathscr{F}\right)$ having supports in $V$. Since $s=0$ on $\Omega_{Q}-\Omega_{f}$, by Proposition 6, p. 235 of [7], there exists a nonnegative integer $m$ such that $f^{m} s=0$ on $\Omega_{Q}$.

(b) The case $p=1, \Gamma\left(\Omega_{Q}, \mathscr{F}\right) \stackrel{\alpha}{\rightarrow} \Gamma\left(\Omega_{Q}-V, \mathscr{F}\right) \stackrel{\beta}{\rightarrow} H_{V}^{1}\left(\Omega_{Q}, \mathscr{F}\right) \rightarrow H^{1}\left(\Omega_{Q}, \mathscr{F}\right)=0$ is exact. $s=\beta\left(s^{*}\right)$ for some $s^{*} \in \Gamma\left(\Omega_{Q}-V, \mathscr{F}\right)$. Since $\Omega_{Q}-V \subset \Omega_{f}$, by Lemma 1 , p. 247 of [7], there exists a nonnegative integer $r$ and $\tilde{s} \in \Gamma\left(\Omega_{Q}, \mathscr{F}\right)$ such that $\tilde{s}=f^{r} s^{*}$ on $\Omega_{Q} \cap \Omega_{f}$. Since $\tilde{s}-f^{r} s^{*}=0$ on $\Omega_{Q_{i}} \cap \Omega_{Q} \cap \Omega_{f}$, by Proposition 6, p. 235 of [7], there exists a nonnegative integer $q_{i}$ such that $f^{q_{i}}\left(\tilde{s}-f^{r} s^{*}\right)=0$ on $\Omega_{Q_{i}} \cap \Omega_{Q}$. Let $q=\max \left(q_{1}, \ldots, q_{k}\right)$. Then $f^{q} \tilde{s}=f^{q+r} s^{*}$ on $\Omega_{Q}-V, f^{q+r} s^{*} \in \operatorname{Im} \alpha$, and $f^{q+r} s=0$.

(c) For the general case, assume $p>1$. Then $H_{V}^{p}\left(\Omega_{Q}, \mathscr{F}\right) \approx H^{p-1}\left(\Omega_{Q}-V, \mathscr{F}\right)$. We identify the two. Let $U_{i}=\Omega_{Q_{i}} \cap \Omega_{Q}$ and $\mathfrak{U}=\left\{U_{i}\right\}_{i=1}^{k}$. $\mathfrak{u}$ covers $\Omega_{Q}-V$. $s$ is represented by an element of $Z^{p-1}(\mathfrak{l}, \mathscr{F})$ given by $s_{i_{0} \cdots i_{p-1}} \in \Gamma\left(U_{i_{0}} \cap \cdots \cap U_{i_{p-1}}, \mathscr{F}\right)$, $1 \leqq i_{0}, \ldots, i_{p-1} \leqq k$. By Lemma 1, p. 247 of [7], there exist a nonnegative integer $r$ and $t_{i_{0} \cdots i_{p-1}} \in \Gamma\left(\Omega_{Q}, \mathscr{F}\right)$ such that $\left(Q_{i_{0}} \cdots Q_{i_{p-1}}\right)^{r} s_{i_{0} \cdots i_{p-1}}=t_{i_{0} \cdots i_{p-1}}$ on $U_{i_{0}} \cap \cdots$ 
$\cap U_{i_{p-1}}$. By Hilbert Nullstellensatz, there exist a nonnegative integer $m$ and $g_{1}, \ldots, g_{k} \in R$ such that $f^{m}=\sum_{i=1}^{k} g_{i} Q_{i}^{r}$. Define $u_{i_{0} \cdots i_{p-2}} \in \Gamma\left(U_{i_{0}} \cap \cdots \cap U_{i_{p-2}}, \mathscr{F}\right)$ by $u_{i_{0}} \cdots i_{p-2}=\sum_{i=1}^{k} g_{i} t_{i_{0} \cdots i_{p-2}}\left(Q_{i_{0}} \cdots Q_{i_{p-2}}\right)^{-r}$. $\left\{u_{i_{0} \cdots i_{p-2}}\right\}$ defines an element $u$ of $C^{p-2}(\mathfrak{U}, \mathscr{F})$. On $U_{i_{0}} \cap \cdots \cap U_{i_{p-1}}$,

$$
\begin{aligned}
(\delta u)_{i_{0}} \cdots i_{p-1} & =\sum_{v=0}^{p-1}(-1)^{v} u_{i_{0}} \cdots i_{v} \cdots i_{p-1} \\
& =\sum_{v=0}^{p-1}(-1)^{v} \sum_{i=1}^{k} g_{i} t_{i i_{0}} \cdots i_{v} \cdots i_{p-1}\left(Q_{i_{0}} \cdots \hat{Q}_{i_{v}} \cdots Q_{i_{p-1}}\right)^{-r} \\
& =\sum_{v=0}^{p-1}(-1)^{v} \sum_{i=1}^{k} g_{i} Q_{i}^{r} s_{i i_{0}} \cdots i_{v} \cdots i_{p-1} \\
& =\sum_{i=1}^{k} g_{i} Q_{i}^{r} \sum_{v=0}^{p-1}(-1)^{v} s_{i i_{0}} \cdots i_{v} \cdots i_{p-1} \\
& =\sum_{i=1}^{k} g_{i} Q_{i}^{r} s_{i_{0}} \cdots i_{p-1}=f^{m} s_{i_{0}} \cdots i_{p-1} .
\end{aligned}
$$

Hence $f^{m_{s}}=0$. Q.E.D.

Lemma 8. Suppose $f_{1}, \ldots, f_{k} \in R$ and $f_{i}$ is not a zero-divisor for $R / \sum_{v=1}^{i-1} R f_{v}$ for $1 \leqq i \leqq k$, where $\sum_{v=1}^{0} R f_{v}=0$. Suppose $I$ is an ideal in $R$ and $\operatorname{dim} I<n-k$. Then there exists $f \in I$ such that $f$ is not a zero-divisor for $R / \sum_{v=1}^{k} R f_{v}$.

Proof. If $R=\sum_{v=1}^{k} R f_{v}$, then there is nothing to prove. Assume $R \neq \sum_{v=1}^{k} R f_{v}$. Since $f_{i}$ is not a zero-divisor for $R / \sum_{v=1}^{i-1} R f_{v}$ for $1 \leqq i \leqq k$, the height of any isolated prime ideal of $\sum_{v=1}^{i} R f_{v}$ is greater than the height of some isolated prime ideal of $\sum_{v=1}^{i-1} R f_{v}$ for $1 \leqq i \leqq k$ [22, Corollary 3, p. 214, Vol. I]. By Theorem 30, p. 240, Vol. I of [22], the height of every isolated prime ideal of $\sum_{v=1}^{k} R f_{v}$ is equal to $k$. By Theorem 20, p. 193, Vol. II of [22], $\operatorname{dim} \sum_{v=1}^{k} R f_{v}=n-k$, because $R$ is simply the polynomial ring of $n$ algebraically independent variables over $C$. Let $\left\{P_{\alpha}\right\}_{\alpha \in A}$ be the set of all associated prime ideals of $\sum_{v=1}^{k} R f_{v}$. By Theorem 26, p. 203, Vol. II of [22], $\operatorname{dim} P_{\alpha}=n-k$ for all $\alpha$. Hence $I \notin P_{\alpha}$ for $\alpha \in A . I \notin \bigcup_{\alpha \in A} P_{\alpha}$ [22, p. 215, Vol. I]. Any element $f$ of $I-\bigcup_{\alpha \in A} P_{\alpha}$ satisfies the requirement [22, Corollary 3, p. 214, Vol. I] Q.E.D.

Proof of Proposition 4. First we prove the following:

If $f_{1}, \ldots, f_{k}, f \in R$ and $f$ is not a zero-divisor

for $R / \sum_{v=1}^{k} R f_{v}$, then the sheaf-homomorphism $\varphi: \mathscr{G} \rightarrow \mathscr{G}$ defined

by multiplication by $f$ is injective, where $\mathscr{G}=\mathcal{O} / \sum_{v=1}^{k} \mathcal{O} f_{v}$.

To prove (15), take arbitrarily $Q \in R$. Suppose $s \in \Gamma\left(\Omega_{Q}, \mathscr{G}\right)$ and $\varphi(s)=0$. By Lemma 1, p. 247 of [7], there exist a nonnegative integer $r$ and $s^{\prime} \in R(\Omega, \mathscr{G})$ such that $s^{\prime}=Q^{r} s$ on $\Omega_{Q} . \varphi\left(s^{\prime}\right)=0$ on $\Omega_{Q}$. By Proposition 6, p. 235 of [7], there exists a nonnegative integer $m$ such that $\varphi\left(Q^{m} s^{\prime}\right)=Q^{m} \varphi\left(s^{\prime}\right)=0$ on $\Omega$. Since $H^{1}\left(\Omega, \sum_{v=1}^{k} O f_{v}\right)$ $=0$ [7, Corollary 1, p. 239], $Q^{m} s^{\prime}$ is the image of some $s^{*} \in R$ under the quotient map $\mathcal{O} \rightarrow \mathscr{G}$. Let $\alpha: \mathcal{O}^{k} \rightarrow \sum_{v=1}^{k} \mathcal{O} f_{v}$ be defined by $\alpha(0, \ldots, 0,1,0, \ldots, 0)=f_{v}$, where 1 is in the $\nu$ th place. Since $H^{1}(\Omega, \operatorname{Ker} \alpha)=0, f_{s}^{*}=\sum_{v=1}^{k} g_{v} f_{v}$ for some 
$g_{1}, \ldots, g_{k} \in R$. Since $f$ is not a zero-divisor for $R / \sum_{v=1}^{k} R f_{v}, s^{*}=\sum_{v=1}^{k} h_{v} f_{v}$ for some $h_{1}, \ldots, h_{k} \in R . Q^{m} s^{\prime}=0$ on $\Omega$, and $s=0$ on $\Omega_{Q}$. (15) is proved.

Fix arbitrarily $Q \in R$. Let $I$ be the ideal of $R$ associated to $V$. For $0 \leqq k \leqq n-r$, consider

If $f_{1}, \ldots, f_{k} \in I$ and $f_{i}$ is not a zero-divisor for $R / \sum_{v=1}^{i-1} R f_{v}$ for

$1 \leqq i \leqq k$ (where $\left.\sum_{v=1}^{0} R f_{v}=0\right)$, then $H_{v}^{p}\left(\Omega_{Q}, \mathcal{O}_{k}\right)=0$ for

$0 \leqq p<n-k-r$, where $\mathcal{O}_{k}=\mathcal{O} / \sum_{v=1}^{k} \mathcal{O} f_{v}$ and $\mathcal{O}_{0}=\mathcal{O}$.

We are going to prove $(16)_{k}$ by descending induction on $k .(16)_{n-r}$ is vacuous. To prove the general case, assume $0 \leqq k<n-r$. Suppose there exists a nonzero element $s$ in $H_{V}^{p}\left(\Omega_{Q}, \mathcal{O}_{k}\right)$ for some $0 \leqq p<n-k-r$. Since $\operatorname{dim} V=r, \operatorname{dim} I=r<n-k$. By Lemma 8, there exists $f \in I$ such that $f$ is not a zero-divisor for $R / \sum_{v=1}^{k} R f_{v}$. By Lemma 7, there exists a nonnegative integer $m$ such that $f^{m} s=0$. Let $f_{k+1}=f^{m}$. By (15) $0 \rightarrow \mathcal{O}_{k} \stackrel{\varphi}{\rightarrow} \mathcal{O}_{k} \rightarrow \mathcal{O}_{k+1} \rightarrow 0$ is exact, where $\varphi$ is defined by multiplication by $f_{k+1}$ and $\mathcal{O}_{k+1}=\mathcal{O} / \sum_{v=1}^{k+1} \mathcal{O} f_{v}$.

$$
H_{V}^{p-1}\left(\Omega_{Q}, \mathcal{O}_{k+1}\right) \longrightarrow H_{V}^{p}\left(\Omega_{Q}, \mathcal{O}_{k}\right) \stackrel{\tilde{\varphi}}{\longrightarrow} H_{V}^{p}\left(\Omega_{Q}, \mathcal{O}_{k}\right)
$$

is exact, where $H_{V}^{-1}\left(\Omega_{Q}, \mathcal{O}_{k+1}\right)=0$. By $(16)_{k+1}, H_{V}^{p-1}\left(\Omega_{Q}, \mathcal{O}_{k+1}\right)=0$. Hence $\tilde{\varphi}$ is injective. $f^{m} s=\tilde{\varphi}(s) \neq 0$. Contradiction. $(16)_{k}$ is true for $0 \leqq k \leqq n-r$.

The proposition follows from (16) 0 . Q.E.D.

In $(\alpha),(\beta),(\gamma)$ and $(\delta)$ below, we suppose the following: $B$ is a ring. $A$ is a subring of $B . B$ is $A$-flat as an $A$-module (Definition 3, p. 34, [8]). $M$ is an $A$-module. $N$ and $N^{\prime}$ are $A$-submodules of $M$. Canonically isomorphic modules are identified.

$(\alpha) N \otimes_{A} B$ can be regarded canonically as a $B$-submodule of $M \otimes_{A} B$ and $(M / N) \otimes_{A} B=\left(M \otimes_{A} B\right) /\left(N \otimes_{A} B\right)$.

$(\alpha)$ follows from tensoring the exact sequence $0 \rightarrow N \rightarrow M \rightarrow M / N \rightarrow 0$ with $B$ over $A$.

( $\beta)\left(N \cap N^{\prime}\right) \otimes_{A} B=\left(N \otimes_{A} B\right) \cap\left(N^{\prime} \otimes_{A} B\right)$.

Let $\varphi: M \rightarrow M / N$ and $\varphi^{\prime}: M \rightarrow M / N^{\prime}$ be the quotient maps and define $\varphi: M \rightarrow$ $(M / N) \oplus\left(M / N^{\prime}\right)$ by $\varphi(f)=\varphi(f) \oplus \varphi^{\prime}(f)$. By tensoring the exact sequence $0 \rightarrow N$ $\cap N^{\prime} \rightarrow M \stackrel{\varphi}{\rightarrow}(M / N) \oplus\left(M / N^{\prime}\right)$ with $B$ over $A$, we obtain the exact sequence $0 \rightarrow\left(N \cap N^{\prime}\right) \otimes_{A} B \rightarrow M \otimes_{A} B \rightarrow\left[(M / N) \oplus\left(M / N^{\prime}\right)\right] \otimes_{A} B$. By $(\alpha), \quad[(M / N) \oplus$ $(M / N)] \otimes_{A} B=\left[\left(M \otimes_{A} B\right) /\left(N \otimes_{A} B\right)\right] \oplus\left[\left(M \otimes_{A} B\right) /\left(N^{\prime} \otimes_{A} B\right)\right]$. On the other hand, the kernel of $M \otimes_{A} B \rightarrow\left[\left(M \otimes_{A} B\right) /\left(N \otimes_{A} B\right)\right] \oplus\left[\left(M \otimes_{A} B\right) /\left(N^{\prime} \otimes_{A} B\right)\right]$ is $\left(N \otimes_{A} B\right) \cap\left(N^{\prime} \otimes_{A} B\right)$. $(\beta)$ follows.

$(\gamma)\left[(N: f)_{M}\right] \otimes_{A} B=\left[\left(N \otimes_{A} B\right): f\right]_{M \otimes_{A} B}$.

Consider the exact sequence $0 \rightarrow(N: f)_{M} \rightarrow M \stackrel{\varphi}{\rightarrow} M / N$, where $\varphi$ is the composite of the quotient map $M \rightarrow M / N$ and the map $M \rightarrow M$ defined by multiplication by $f$. Tensoring it with $B$ over $A$ yields the exact sequence $0 \rightarrow\left[(N: f)_{M}\right] \otimes_{A} B \rightarrow$ $M \otimes_{A} B \rightarrow(M / N) \otimes_{A} B$. By $(\alpha),(M / N) \otimes_{A} B=\left(M \otimes_{A} B\right) /\left(N \otimes_{A} B\right)$. On the other hand, the kernel of $M \otimes_{A} B \rightarrow\left(M \otimes_{A} B\right) /\left(N \otimes_{A} B\right)$ is $\left(\left[N \otimes_{A} B\right]: f\right)_{M \otimes_{A} B}$. $(\gamma)$ follows. 
( $\delta$ ) If $I$ is a finitely generated ideal in $A$, then

$$
\left[(N: I)_{M}\right] \otimes_{A} B=\left(\left[N \otimes_{A} B\right]:\left[I \otimes_{A} B\right]\right)_{M \otimes_{A} B} .
$$

Suppose $I=\sum_{i=1}^{k} A f_{i} . \quad(N: I)_{M}=\bigcap_{i=1}^{k}\left(N: f_{i}\right)_{M}$ and $\left(\left[N \otimes_{A} B\right]:\left[I \otimes_{A} B\right]\right)_{M \otimes_{A} B}$ $=\bigcap_{i=1}^{k}\left(\left[N \otimes_{A} B\right]: f_{i}\right)_{M \otimes_{A} B} .(\delta)$ follows from $(\beta)$ and $(\gamma)$.

Proposition 5. Suppose $A$ is a Noetherian subring of a Noetherian ring $B$ and $B$ is $A$-flat as an A-module. Suppose $I$ is an ideal in $A$ and $N$ is an A-submodule of a finitely generated A-module $M$. Then

$$
\left[\bigcup_{k=1}^{\infty}\left(N: I^{k}\right)_{M}\right] \otimes_{A} B=\bigcup_{k=1}^{\infty}\left(\left[N \otimes_{A} B\right]:\left[I \otimes_{A} B\right]^{k}\right)_{M \otimes_{A} B}
$$

Proof. $(N: I)_{M} \subset\left(N: I^{2}\right)_{M} \subset \ldots \subset\left(N: I^{k}\right)_{M} \subset \ldots$ and

$$
\begin{aligned}
\left(\left[N \otimes_{A} B\right]:\left[I \otimes_{A} B\right]\right)_{M \otimes_{A} B} & \subset\left(\left[N \otimes_{A} B\right]:\left[I \otimes_{A} B\right]^{2}\right)_{M \otimes_{A} B} \subset \ldots \\
& \subset\left(\left[N \otimes_{A} B\right]:\left[I \otimes_{A} B\right]^{k}\right)_{M \otimes_{A} B} \subset \ldots
\end{aligned}
$$

are both eventually stationary, because $M$ is finitely generated over the Noetherian ring $A$ and $M \otimes_{A} B$ is finitely generated over the Noetherian ring $B$. For some $l \geqq 1, \bigcup_{k=1}^{\infty}\left(N: I^{k}\right)_{M}=\left(N: I^{l}\right)_{M}$ and

$$
\bigcup_{k=1}^{\infty}\left(\left[N \otimes_{A} B\right]:\left[I \otimes_{A} B\right]^{k}\right)_{M \otimes_{A} B}=\left(\left[N \otimes_{A} B\right]:\left[I \otimes_{A} B\right]^{l}\right)_{M \otimes_{A} B}
$$

The lemma follows from $(\delta)$. Q.E.D.

In $(\varepsilon),(\zeta)$ and $(\eta)$ below, we assume the following: $(A, B)$ is a flat couple of local rings [8, Definition 4, p. 36]. $M$ is an $A$-module. $\tilde{M}=M \otimes_{A} B$. Since the natural map $M \rightarrow \tilde{M}$ is injective [8, Proposition 22, p. 36], $M$ is identified canonically as a subset of $\tilde{M}$.

(e) If $f \in A$ is not a zero-divisor for $M$, then $f$ is not a zero-divisor for $\tilde{M}$.

Since the homomorphism $M \rightarrow M$ defined by multiplication by $f$ is injective and $B$ is $A$-flat, the homomorphism $\tilde{M} \rightarrow \tilde{M}$ defined by multiplication by $f$ is injective. $(\varepsilon)$ follows.

(ל) If $I$ is an ideal in $A$, then $(I \tilde{M}) \cap M=I M$.

$(\zeta)$ is the consequence of $(M / I M) \otimes_{A} B=\tilde{M} / I \tilde{M}$ and the injectivity of the canonical homomorphism $M / I M \rightarrow(M / I M) \otimes_{A} B$ [8, Proposition 22, p. 36].

$(\eta)$ If $f_{1}, \ldots, f_{k}$ are in the intersection of the maximal ideal of $A$ and $B$ and $f_{1}, \ldots, f_{k}$ form an $M$-sequence $\left[4\right.$, p. 94], then $f_{1}, \ldots, f_{k}$ form an $\tilde{M}$-sequence.

For $1 \leqq i \leqq k, f_{i}$ is not a zero-divisor for $M / \sum_{v=1}^{i-1} f_{v} M$. By $(\varepsilon), f_{i}$ is not a zerodivisor for $\left(M / \sum_{v=1}^{i-1} f_{v} M\right) \otimes_{A} B=\tilde{M} / \sum_{v=1}^{i-1} f_{v} \tilde{M}$ for $1 \leqq i \leqq k$. ( $\eta$ follows.

Proposition 6. Suppose $(A, \mathfrak{m})$ and $(B, \mathfrak{n})$ are local rings such that $(A, B)$ is a flat couple and $\mathfrak{m} B=\mathfrak{n}$. If $M$ is a finitely generated $A$-module and $\tilde{M}=M \otimes_{A} B$, then $\operatorname{codh}_{A} \tilde{M}=\operatorname{codh}_{B} \tilde{M}$.

Proof. Let $\operatorname{codh}_{A} M=k$. Let $f_{1}, \ldots, f_{k}$ be an $M$-sequence. (In the case $k=0$, $f_{1}, \ldots, f_{k}$ is just the empty sequence and the argument that follows applies also to this case.) By $(\eta), f_{1}, \ldots, f_{k}$ is an $\tilde{M}$-sequence. We need only prove that $f_{1}, \ldots, f_{k}$ is a maximal $\tilde{M}$-sequence. 
Since $f_{1}, \ldots, f_{k}$ is a maximal $M$-sequence, every element of $\mathfrak{m}$ is a zero-divisor for $M / \sum_{v=1}^{k} f_{v} M . \mathfrak{m}$ is an associated prime ideal of the $A$-submodule $\sum_{v=1}^{k} f_{v} M$ of the $A$-module $M$. There exist $g \in M$ and a natural number $r$ such that $g \notin \sum_{v=1}^{k} f_{v} M$

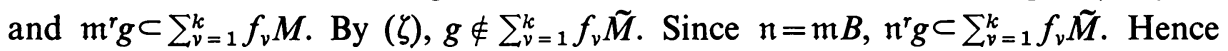
$\mathfrak{n}$ is an associated prime ideal of the $B$-submodule $\sum_{v=1}^{k} f_{v} \tilde{M}$ of the $B$-module $\tilde{M}$. Every element of $\mathfrak{n}$ is a zero-divisor for $\tilde{M} / \sum_{v=1}^{k} f_{v} \tilde{M} . f_{1}, \ldots, f_{k}$ is a maximal $\tilde{M}$-sequence. Q.E.D.

Proposition 7. Suppose $X$ is a complex algebraic space, $V$ is an algebraic subvariety of $X$, and $\mathscr{F}$ is a coherent algebraic sheaf on $X$. Then the canonical homomorphism $\mathscr{H}_{v}^{0}(\mathscr{F})^{h} \rightarrow \mathscr{H}_{v}^{0}\left(\mathscr{F}^{h}\right)$ is an isomorphism.

Proof. It is clear from the definitions that $\mathscr{H}_{v}^{0}(\mathscr{F})=O[V]_{\mathscr{F}}$ and $\mathscr{H}_{v}^{0}\left(\mathscr{F}^{h}\right)$ $=O[V]_{F^{n}}$.

Let $\mathcal{O}$ be the algebraic structure-sheaf of $X$ and $\mathscr{H}$ be the associated analytic structure-sheaf. Let $\mathscr{I}$ be the algebraic ideal-sheaf of $V$. Then $\mathscr{I}^{h}$ is the analytic ideal-sheaf of $V$ [8, Proposition 4, p. 10]. By [10, Theorem 1, p. 376], $\mathcal{O}[V]_{\mathscr{F}}$ $=\bigcup_{k=1}^{\infty}\left(O:\left(\mathscr{I}^{h}\right)^{k}\right)_{\mathscr{F}}$. Analogously it can be proved that $O[V]_{\mathscr{F}}=\bigcup_{k=1}^{\infty}\left(O: \mathscr{I}^{k}\right)_{\mathscr{F}}$.

For every $x \in X,\left(\mathcal{O}_{x}, \mathscr{H}_{x}\right)$ is a flat couple [8, Corollary 1, p. 11]. The proposition follows from Proposition 5. Q.E.D.

Proof of Theorem B. Let $\mathcal{O}$ be the algebraic structure-sheaf of $X$ and let $\mathscr{H}$ be the associated analytic structure-sheaf. Since $\left(\mathcal{O}_{x}, \mathscr{H}_{x}\right)$ is a flat couple for every $x \in X, S_{k}(\mathscr{F} \mid X-V)=S_{k}\left(\mathscr{F}^{h} \mid X-V\right)$ for every $k \geqq 0$ by Proposition 6 . Hence the equivalence of the coherence of $\mathscr{H}_{V}^{o}(\mathscr{F}), \ldots, \mathscr{H}_{V}^{q+1}(\mathscr{F})$ and the coherence of $\mathscr{H}_{v}^{0}\left(\mathscr{F}^{h}\right), \ldots, \mathscr{H}_{v}^{q+1}\left(\mathscr{F}^{h}\right)$ follows from Theorems A and $\mathrm{A}^{\prime}$.

Suppose we have the coherence of either $\mathscr{H}_{v}^{0}(\mathscr{F}), \ldots, \mathscr{H}_{V}^{a+1}(\mathscr{F})$ or $\mathscr{H}_{v}^{0}\left(\mathscr{F}^{h}\right), \ldots$, $\mathscr{H}_{v}^{q+1}\left(\mathscr{F}^{h}\right)$ (and hence the coherence of all). We are going to prove the bijectivity of $\mathscr{H}_{v}^{k}(\mathscr{F})^{h} \rightarrow \mathscr{H}_{v}^{k}\left(\mathscr{F}^{h}\right)$ for $0 \leqq k \leqq q+1$ by induction on $k$. The case $k=0$ follows from Proposition 7. For the general case fix $0<k \leqq q+1$.

Let $\mathscr{K}=O[V]_{\mathscr{F}}$ and $\theta: X-V \rightarrow X$ be the inclusion map. Since Supp $\mathscr{K} \subset V$, $\theta_{l}(\mathscr{K})=0$ for $l \geqq 0$. Hence $H_{V}^{1}(\mathscr{K}) \approx \operatorname{Coker}\left(\mathscr{K} \rightarrow \theta_{0}(\mathscr{K})\right)=0$ and $H_{V}^{l}(\mathscr{K}) \approx \theta_{l-1}(\mathscr{K})$ $=0$ for $l>1$. The exact sequence $\mathscr{H}_{v}^{l}(\mathscr{K}) \rightarrow \mathscr{H}_{v}^{l}(\mathscr{F}) \rightarrow \mathscr{H}_{v}^{l}(\mathscr{F} / \mathscr{K}) \rightarrow \mathscr{H}_{v}^{l+1}(\mathscr{K})$ implies that $\mathscr{H}_{v}^{l}(\mathscr{F}) \approx \mathscr{H}_{v}^{l}(\mathscr{F} / \mathscr{K})$ for $l \geqq 1$. Likewise we have $\mathscr{H}_{v}^{l}\left(\mathscr{F}^{h}\right) \approx \mathscr{H}_{V}^{l}\left((\mathscr{F} / \mathscr{K})^{h}\right)$ for $l \geqq 1$. Hence, by replacing $\mathscr{F}$ by $\mathscr{F} / \mathscr{K}$, we can assume that $O[V]_{\mathscr{F}}=0$.

We can assume that $X$ is an algebraic subspace of some $C^{N}$. Choose $f \in \Gamma(X, \mathcal{O})$ such that (i) $f$ vanishes identically on $V$ and (ii) for any $l \geqq 0, f$ does not vanish identically on any branch of Supp $O_{[1] \mathscr{F}}$ not contained in $V$. Let $\varphi: \mathscr{F} \rightarrow \mathscr{F}$ be defined by multiplication by $f$. Ker $\varphi=0$ on $X-V$ (algebraic analog of Lemma 3). Since $O[V]_{\mathscr{F}}=0, \operatorname{Ker} \varphi=0$ on $X$.

Let $Q$ be an arbitrary relatively compact open subset of $X$. Since $\mathscr{H}_{V}^{k}(\mathscr{F})$ and $\mathscr{H}_{V}^{k}\left(\mathscr{F}^{h}\right)$ are coherent and $f$ vanishes identically on their supports, there exists a natural number $m$ such that $f^{m} \mathscr{H}_{V}^{k}(\mathscr{F})=0$ on $X$ and $f^{m} \mathscr{H}_{V}^{k}\left(\mathscr{F}^{h}\right)=0$ on $Q$. 
Let $\psi=\varphi \circ \cdots \circ \varphi(m$ times $)$. Let $\mathscr{G}=$ Coker $\psi$. Then

$$
S_{l+q}(\mathscr{G} \mid X-V) \subset S_{l+q+1}(\mathscr{F} \mid X-V) .
$$

Hence $\mathscr{H}_{V}^{0}(\mathscr{G}), \ldots, \mathscr{H}_{V}^{q}(\mathscr{G})$ and $\mathscr{H}_{V}^{0}\left(\mathscr{G}^{h}\right), \ldots, \mathscr{H}_{V}^{q}\left(\mathscr{G}^{h}\right)$ are coherent by Theorems A and $\mathrm{A}^{\prime}$.

The exact sequence $0 \rightarrow \mathscr{F} \stackrel{\Downarrow}{\rightarrow} \mathscr{F} \rightarrow \mathscr{G} \rightarrow 0$ yields the following commutative diagram with exact rows:

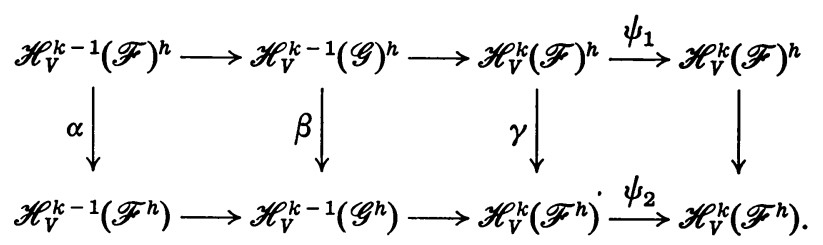

$\operatorname{Im} \psi_{1}=0$ on $X$ and $\operatorname{Im} \psi_{2}=0$ on $Q$. By induction hypothesis, $\alpha$ and $\beta$ are isomorphisms. Hence $\gamma$ is isomorphic on $Q$. The induction process is complete, because $Q$ is arbitrary. Q.E.D.

Finally, we give an interesting relation between the Ext functor and direct images in a very special case (Proposition 8 below).

LemMA 9. Suppose $R$ is a ring, $F$ is an $R$-module, and

$$
R^{p_{q+1}} \stackrel{\varphi_{q}}{\longrightarrow} R^{p_{q}} \stackrel{\varphi_{q-1}}{\longrightarrow} \cdots \stackrel{\varphi_{1}}{\longrightarrow} R^{p_{1}} \stackrel{\varphi_{0}}{\longrightarrow} R^{p_{0}} \stackrel{\varepsilon}{\longrightarrow} F \longrightarrow 0
$$

is an exact sequence. Suppose

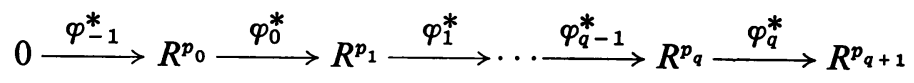

is obtained by applying the functor $\operatorname{Hom}_{R}(\cdot, R)$ to

$$
R^{p_{q+1}} \stackrel{\varphi_{q}}{\longrightarrow} R^{p_{q}} \stackrel{\varphi_{q-1}}{\longrightarrow} \cdots \stackrel{\varphi_{1}}{\longrightarrow} R^{p_{1}} \stackrel{\varphi_{0}}{\longrightarrow} R^{p_{0}} \longrightarrow 0 .
$$

Then $\operatorname{Ext}_{R}^{k}(F, R) \approx \operatorname{Ker} \varphi_{k}^{*} / \operatorname{Im} \varphi_{k-1}^{*}$ for $0 \leqq k \leqq q$.

Proof. Fix $0 \leqq k \leqq q$. We are going to prove $\operatorname{Ext}_{R}^{k}(F, R) \approx \operatorname{Ker} \varphi_{k}^{*} / \operatorname{Im} \varphi_{k-1}^{*}$ by induction on $k$. The case $k=0$ follows from the left-exactness of the functor $\operatorname{Hom}_{R}(\cdot, R)$.

Let $G=\operatorname{Im} \varphi_{0}$. The following commutative diagram has exact rows and exact columns:

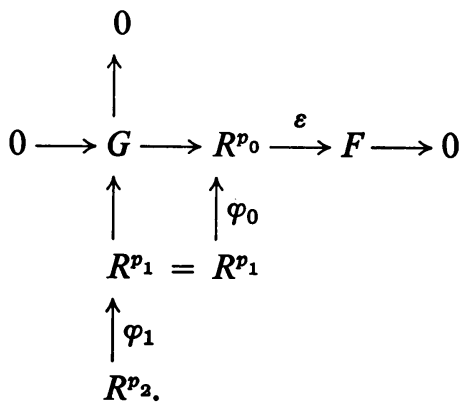


By applying the functor $\operatorname{Hom}_{R}(\cdot, R)$, we obtain the following commutative diagram with exact rows and exact columns:

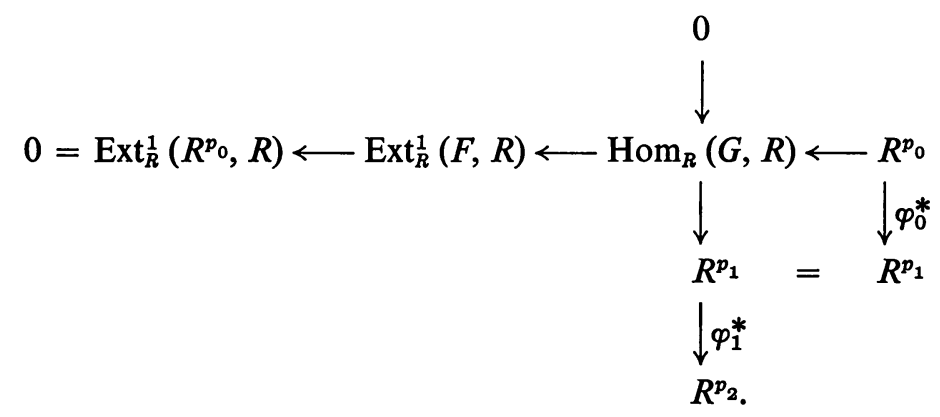

We see readily that $\operatorname{Ext}_{R}^{1}(F, R) \approx \operatorname{Ker} \varphi_{1}^{*} / \operatorname{Im} \varphi_{0}^{*}$. The case $k=1$ is proved.

For the general case, assume $k>1$. The exact sequence $0 \rightarrow G \rightarrow R^{p_{0}} \rightarrow F \rightarrow 0$ yields the exact sequence $0=\operatorname{Ext}_{R}^{k-1}\left(R^{p_{0}}, R\right) \rightarrow \operatorname{Ext}_{R}^{k-1}(G, R) \rightarrow \operatorname{Ext}_{R}^{k}(F, R) \rightarrow$ $\operatorname{Ext}_{R}^{k}\left(R^{p_{0}}, R\right)=0$. By induction hypothesis, $\operatorname{Ext}_{R}^{k-1}(G, R) \approx \operatorname{Ker} \varphi_{k}^{*} / \operatorname{Im} \varphi_{k-1}^{*}$. Hence the general case follows. Q.E.D.

Proposition 8. Suppose $G$ is an open subset of $C^{n}, V$ is an analytic subvariety of dimension $r$ in $G$, and $\theta: G-V \rightarrow G$ is the inclusion map. Suppose $\mathscr{F}$ is a coherent analytic sheaf on $G$ such that $\mathscr{F}$ is locally free on $G-V$. Then $\theta_{k}(\mathscr{F} \mid G-V)$ $\approx \operatorname{Ext}_{\mathscr{H}}^{k}\left(\operatorname{Hom}_{\mathscr{H}}(\mathscr{F}, \mathscr{H})\right)$ for $0 \leqq k \leqq n-r-2$, where $\mathscr{H}$ is the analytic structuresheaf of $C^{n}$.

Proof. Fix $0 \leqq k \leqq n-r-2$. We are going to prove that $\theta_{k}(\mathscr{F} \mid G-V) \approx$ $\operatorname{Ext}_{\mathscr{H}}^{k}\left(\operatorname{Hom}_{\mathscr{H}}(\mathscr{F}, \mathscr{H})\right)$. Let $\mathscr{G}=\operatorname{Hom}_{\mathscr{H}}(\mathscr{F}, \mathscr{H})$ and $\mathscr{G}^{*}=\operatorname{Hom}_{\mathscr{H}}(\mathscr{G}, \mathscr{H})$. Since the problem is local in nature, we can assume that we have the following exact sequence on $G$ :

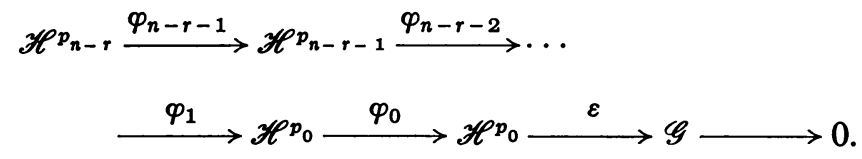

By applying the functor $\operatorname{Hom}_{\mathscr{H}}(\cdot, \mathscr{H})$, we obtain

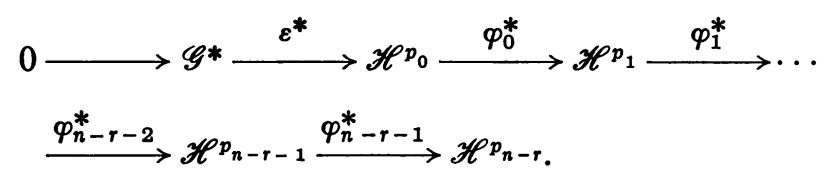

Since $\mathscr{F}$ is locally free on $G-V,(17)$ is exact on $G-V$ and $\mathscr{F} \approx \mathscr{G}^{*}$ on $G-V$. The following commutative diagram with exact rows

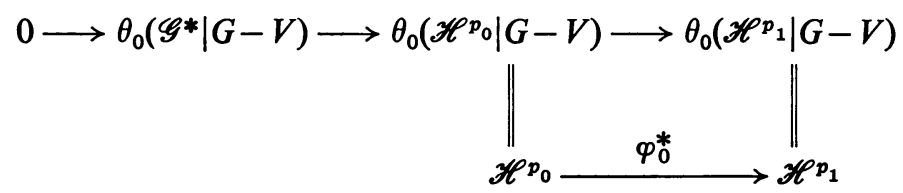


implies that $\theta_{0}(\mathscr{F} \mid G-V) \approx \operatorname{Ker} \varphi_{0}^{*}$. Hence the case $k=0$ follows from Lemma 9 .

For the general case, assume $0<k \leqq n-r-2$. The exact sequence $0 \rightarrow \operatorname{Ker} \varphi_{v}^{*}$ $\rightarrow \mathscr{H}^{p_{v}} \rightarrow \operatorname{Ker} \varphi_{v+1}^{*} \rightarrow 0$ on $G-V$ yields the exact sequence $0=\theta_{k-v-1}\left(\mathscr{H}^{p_{v}} \mid G-V\right)$ $\rightarrow \theta_{k-v-1}\left(\operatorname{Ker} \varphi_{v+1}^{*} \mid G-V\right) \rightarrow \theta_{k-v}\left(\operatorname{Ker} \varphi_{v}^{*} \mid G-V\right) \rightarrow \theta_{k-v}\left(\mathscr{H}^{p_{v}} \mid G-V\right)=0$ for $0 \leqq \nu$ $\leqq k-2$. Hence $\theta_{k}(\mathscr{F} \mid G-V) \approx \theta_{k}\left(\mathscr{G}^{*} \mid G-V\right) \approx \theta_{k}\left(\operatorname{Ker} \varphi_{0}^{*} \mid G-V\right) \approx \theta_{k-1}\left(\operatorname{Ker} \varphi_{1}^{*} \mid G-V\right)$ $\approx \cdots \approx \theta_{1}\left(\operatorname{Ker} \varphi_{k-1}^{*} \mid G-V\right)$.

Since the following commutative diagram has exact rows and exact columns on $G-V$

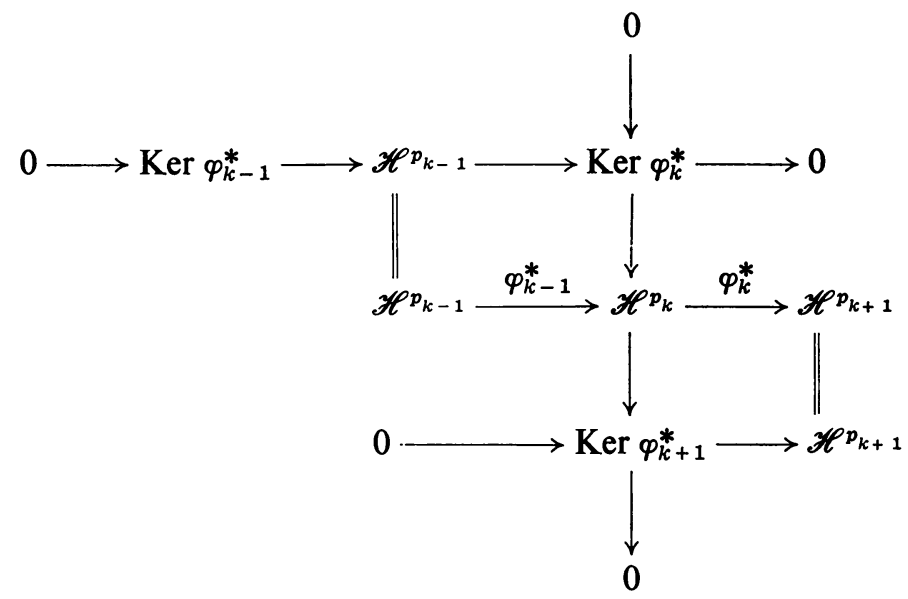

the first and third rows and the columns of the following commutative diagram are exact

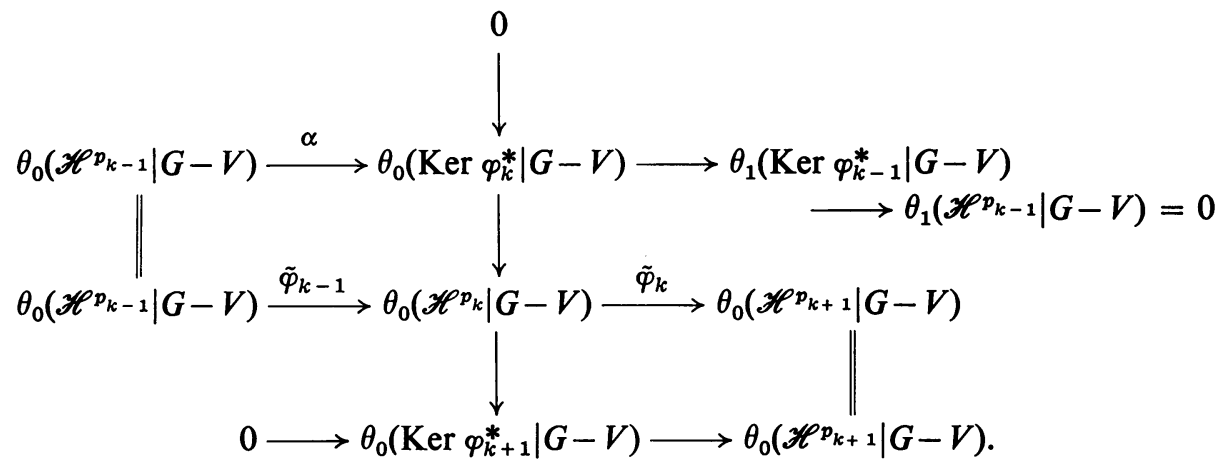

Hence $\theta_{0}\left(\operatorname{Ker} \varphi_{k}^{*} \mid G-V\right) \approx \operatorname{Ker} \tilde{\varphi}_{k}, \operatorname{Im} \alpha \approx \operatorname{Im} \tilde{\varphi}_{k-1}$, and

$$
\theta_{1}\left(\operatorname{Ker} \varphi_{k-1}^{*} \mid G-V\right) \approx \theta_{0}\left(\operatorname{Ker} \varphi_{k}^{*} \mid G-V\right) / \operatorname{Im} \alpha .
$$

Since $\theta_{0}\left(\mathscr{H}^{p_{v}} \mid G-V\right) \approx \mathscr{H}^{p_{v}}$ for $\nu=k-1, k, k+1$, we have $\tilde{\varphi}_{v}=\varphi_{v}^{*}$ for $\nu=k-1, k$. Therefore $\theta_{k}(\mathscr{F} \mid G-V) \approx \theta_{1}\left(\operatorname{Ker} \varphi_{k-1}^{*} \mid G-V\right) \approx \operatorname{Ker} \tilde{\varphi}_{k} / \operatorname{Im} \tilde{\varphi}_{k-1}=\operatorname{Ker} \varphi_{k}^{*} / \operatorname{Im} \varphi_{k-1}^{*}$. By Lemma $9, \operatorname{Ker} \varphi_{k}^{*} / \operatorname{Im} \varphi_{k-1}^{*} \approx \operatorname{Ext}_{\mathscr{H}}^{k}(\mathscr{G}, \mathscr{H})=\operatorname{Ext}_{\mathscr{H}}^{k}\left(\operatorname{Hom}_{\mathscr{H}}(\mathscr{F}, \mathscr{H}), \mathscr{H}\right)$. Q.E.D.

After the submission of this paper, I learned that G. Trautmann simultaneously and independently had also obtained the equivalence of (i) and (iii) of Theorem A in Invent. Math. 8 (1969), 143-174. 


\section{REFERENCES}

1. J. Frisch and J. Guenot, Prolongement de faisceaux analytiques cohérents, Invent. Math. 7 (1969), 321-343.

2. A. Grothendieck, Cohomologie locale des faisceaux cohérents et théorèmes de Lefschetz locaux et globaux, Séminaire de Géométrie Algébrique, Inst. Hautes Études Sci., Paris, 1962. MR 35 \#1604.

3. R. Hartshome, Local cohomology, Springer-Verlag, Berlin, 1967. MR 37 \#219.

4. M. Nagata, Local rings, Interscience, New York, 1962. MR 27 \#5790.

5. G. Scheja, Riemannsche Hebbarkeitssätze für Cohomologieklassen, Math. Ann. 144 (1961), 345-360. MR 26 \#6437.

6. - Fortsetzungssätze der komplex-analytischen Cohomologie und ihre algebraische Charakterisierung, Math. Ann. 157 (1964), 75-94. MR 31 \#738.

7. J.-P. Serre, Faisceaux algébriques cohérents, Ann. of Math. 157 (1964), 197-278.

8. - Géométrie algébrique et géométrie analytique, Ann. Inst. Fourier Grenoble 6 (1955/56), 1-42. MR 18, 511.

9. - Prolongement de faisceaux analytiques cohérents, Ann. Inst. Fourier Grenoble 16 (1966), fasc. 1, 363-374. MR 35 \#3088.

10. Y.-T. Siu, Noether-Lasker decomposition of coherent analytic subsheaves, Trans. Amer. Math. Soc. 135 (1969), 375-385. MR 38 \#2340.

11. - Extension of locally free analytic sheaves, Math. Ann. 179 (1969), 285-294.

12. - Absolute gap-sheaves and extensions of coherent analytic sheaves, Trans. Amer. Math. Soc. 141 (1969), 361-376.

13. - Extending coherent analytic sheaves through subvarieties, Bull. Amer. Math. Soc. 75 (1969), 123-126.

14. —- Extending coherent analytic sheaves, Ann. of Math. 90 (1969), 108-143.

15. _- Analytic sheaves of local cohomology, Bull. Amer. Math. Soc. 75 (1969), 10111012.

16. W. Thimm, Lückengarben von kohärenten analytischen Modulgarben, Math. Ann. 148 (1962), 372-394. MR 26 \#1493.

17. - Struktur- und Singularitätsuntersuchungen an kohärenten analytischen Modulgarben, J. Reine Angew. Math. 234 (1969), 123-151.

18. G. Trautmann, Ein Kontinuitätssatz für die Fortsetzung kohärenter analytischer Garben, Arch. Math. 18 (1967), 188-196. MR 35 \#6869.

19. - Abgeschlossenheit von Corandmoduln und Fortsetzbarkeit kohärenter analytischer Garben, Invent. Math. 4 (1968), 216-230. MR 37 \#4289.

20. —, Fortsetzung lokal-freier Garben über 1-dimensionale Singularitätenmengen, Ann. Scuola Norm. Sup. Pisa 23 (1969), 155-184.

21. - Cohérence des faisceaux analytiques de la cohomologie local, C. R. Acad. Sci. Paris 267 (1968), 694-695.

22. O. Zariski and P. Samuel, Commutative algebra. Vols. I, II, Van Nostrand, Princeton, N. J., 1958, 1960. MR 19, 833. MR 22 \#11006.

\section{UNIVERSITY OF NOTRE DAME,} Notre DAME, INDIANA 46556 